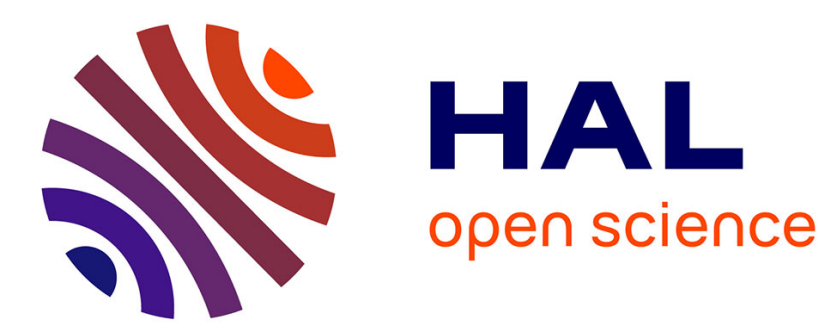

\title{
DYNAMICAL GENERALIZATIONS OF THE LAGRANGE SPECTRUM
}

\author{
Sébastien Ferenczi
}

\section{To cite this version:}

Sébastien Ferenczi. DYNAMICAL GENERALIZATIONS OF THE LAGRANGE SPECTRUM. Journal d'analyse mathématique, 2012. hal-01265514

\section{HAL Id: hal-01265514 \\ https://hal.science/hal-01265514}

Submitted on 1 Feb 2016

HAL is a multi-disciplinary open access archive for the deposit and dissemination of scientific research documents, whether they are published or not. The documents may come from teaching and research institutions in France or abroad, or from public or private research centers.
L'archive ouverte pluridisciplinaire HAL, est destinée au dépôt et à la diffusion de documents scientifiques de niveau recherche, publiés ou non, émanant des établissements d'enseignement et de recherche français ou étrangers, des laboratoires publics ou privés. 


\title{
DYNAMICAL GENERALIZATIONS OF THE LAGRANGE SPECTRUM
}

\author{
SÉBASTIEN FERENCZI
}

\begin{abstract}
We compute two invariants of topological conjugacy, the upper and lower limits of the inverse of Boshernitzan's $n e_{n}$, where $e_{n}$ is the smallest measure of a cylinder of length $n$, for three families of symbolic systems, the natural codings of rotations and three-interval exchanges and the Arnoux-Rauzy systems. The sets of values of these invariants for a given family of systems generalize the Lagrange spectrum, which is what we get for the family of rotations with the upper limit of $\frac{1}{n e_{n}}$.
\end{abstract}

The Lagrange spectrum is the set of finite values of $L(\alpha)$ for all irrational numbers $\alpha$, where $L(\alpha)$ is the largest constant $c$ such that $\left|\alpha-\frac{p}{q}\right| \leq \frac{1}{c q^{2}}$ for infinitely many integers $p$ and $q$ (a variant is known as the Markov spectrum, see Section 1.3 below). It was recently remarked that this arithmetic definition can be replaced by a dynamical definition involving the irrational rotations of angle $\alpha$, through their natural coding by the partition $\{[0,1-$ $\alpha[,[1-\alpha, 1[\}$. Namely, as we prove in Theorem 2.4 below which was never written before, $L(\alpha)$ is also the upper limit of the inverse of the so-called Boshernitzan's ne $e_{n}$, where $e_{n}$ is the smallest (Lebesgue) measure of the nonempty cylinders of length $n$.

Thus, for any symbolic dynamical system, it is interesting to compute two new invariants of topological conjugacy, $\lim \sup _{n \rightarrow+\infty} \frac{1}{n e_{n}}$ and $\liminf _{n \rightarrow+\infty} \frac{1}{n e_{n}}$. Moreover, for a given family of systems, the set of all values of these invariants can be called the upper, resp. lower $B L$ (for Boshernitzan and Lagrange) spectrum. In this paper, we compute these spectra for three families of systems: the irrational rotations (seen as two-interval exchanges), the three-interval exchanges, both coded by the natural partition of the interval generated by the discontinuities, and the Arnoux-Rauzy systems. In each of these cases, we use an induction (or renormalization) process, which is respectively a variant of the Euclid algoritm, the selfdual induction of [20], and the natural one defined in [3]. A multiplicative form of the process yields explicit formulas for our invariants, and these formulas are then exploited in each case by using the underlying algorithm of approximation of real numbers by rationals, which is respectively the classical continued fraction expansion, an extension of a semi-regular continued fraction expansion, and the algorithm which motivated the study of Arnoux-Rauzy systems.

What we get in the end is a first partial description of the five new sets we introduced beside the classical Lagrange spectrum. For rotations, the lower BL spectrum is a compact

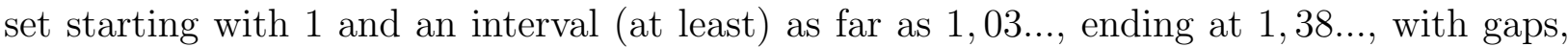
above an accumulation point at 1,23... For three-interval exchanges, the upper BL spectrum looks, perhaps deceptively, like two times the Lagrange spectrum, starting at $2 \sqrt{5}$ with gaps and an accumulation point at 6 , and ending with an interval (at least) from $14,8 \ldots$ to infinity; the lower BL spectrum is fully determined and is none other than the interval $[2,+\infty]$. For

Date: October 18, 2011.

1991 Mathematics Subject Classification. 37B10, 11J06, 68R15. 
Arnoux-Rauzy systems, we deal with cubic numbers and our knowledge is only embryonic: the upper BL spectrum starts at $8,44 \ldots$, with gaps, and ends at infinity, the lower BL spectrum starts at 2, ends at infinity, and contains at least all the integers as accumulation points.

As a consequence, we get new uniquely ergodic systems for which $n e_{n}$ does not tend to zero when $n$ tends to infinity, showing that Boshernitzan's criterion (see Section 1.4) is not a necessary condition; their existence in the family of three-interval exchanges was known but a proof was never written, while the examples in the family of Arnoux-Rauzy systems are new, and surprising as these systems are often thought to behave like rotations, see the discussion at the end of Section 4. An interesting open problem would be to compute the BL spectra of the family of all uniquely ergodic symbolic systems, see Section 5.

\section{Preliminaries}

\subsection{Languages.}

Definition 1.1. We look at finite words on a finite alphabet $\mathcal{A}$. A word with $r$ letters, $w_{1} \ldots w_{r}$, is of length $r$. The concatenation of two words $w$ and $w^{\prime}$ is denoted by $w w^{\prime}$. The empty word is the unique word of length zero.

$A$ word $w=w_{1} \ldots w_{r}$ occurs at place $i$ in a word $v=v_{1} \ldots v_{s}$ or an infinite sequence $v=v_{1} v_{2} \ldots$ if $w_{1}=v_{i}, \ldots, w_{r}=v_{i+r-1}$. We say that $w$ is a factor of $v$. The empty word is a factor of any $v$. Prefixes and suffixes are defined in the usual way.

$A$ language $L$ is a set of words such if $w$ is in $L$, all its factors are in $L$, aw is in $L$ for at least one letter a of $\mathcal{A}$, and $w b$ is in $L$ for at least one letter $b$ of $\mathcal{A}$.

A language $L$ is uniformly recurrent if for each $w$ in $L$ there exists $n$ such that $w$ occurs in each word of length $n$ of $L$.

A language $L$ is now fixed.

Definition 1.2. A word $w$ is right special, resp. left special if there exist at least two different letters $x$ such that $x w$, resp. $w x$, is in L. If $w$ is both right special and left special, $w$ is bispecial.

The complexity of $L$ is the function $p_{L}$ which to each positive integer $n$ associates the number of different words of length $n$ in $L$.

The Rauzy graph of length $n$ of $L$ is the graph whose vertices are the words of length $n$ in $L$, with an edge $w \rightarrow w^{\prime}$ if there exists a word $v$ of length $n-1$ such that $w=a v, w^{\prime}=v b$, and $a v b \in L$.

\subsection{Symbolic dynamics.}

Definition 1.3. The symbolic dynamical system associated to a language $L$ is the one-sided shift $S\left(x_{0} x_{1} x_{2} \ldots\right)=x_{1} x_{2} \ldots$ on the subset $X_{L}$ of $\mathcal{A}^{\mathbb{N}}$ made with the infinite sequences such that for every $r<s, x_{r} \ldots x_{s}$ is in $L$.

For a word $w=w_{1} \ldots w_{r}$ in $L$, the cylinder $[w]$ is the set $\left\{x \in X_{L} ; x_{0}=w_{1}, \ldots x_{r-1}=w_{r}\right\}$. $\left(X_{L}, S\right)$ is minimal if $L$ is uniformly recurrent.

$\left(X_{L}, S\right)$ is uniquely ergodic if there is one $S$-invariant probability measure $\mu$; then the frequency of the word $w$ is the measure $\mu[w]$.

Starting from a (in general, geometric in origin) topological dynamical system $(X, T)$, we can get a symbolic dynamical system: 
Definition 1.4. For a transformation $T$ defined on a set $X$, partitioned into $X_{1}, \ldots X_{r}$, and a point $x$ in $X$, its trajectory is the infinite sequence $\left(x_{n}\right)_{n \in \mathbb{N}}$ defined by $x_{n}=i$ if $T^{n} x$ falls into $X_{i}, 1 \leq i \leq r$.

The language $L(T)$ is the set of all finite factors of its trajectories.

The coding of $(X, T)$ by the partition $\left\{X_{1}, \ldots X_{r}\right\}$ is the symbolic dynamical system $\left(X_{L(T)}, S\right)$.

We shall often assimilate a dynamical system $(X, T)$ with its coding by a partition. Note that the word $w_{1} \ldots w_{n}$ has length $n$; in most cases, the cylinder $\left[w_{1} \ldots w_{n}\right]$ is assimilated to an interval $J$, on which $\mu$ is the Lebesgue measure; the (geometrical) length of $J$ is also the Lebesgue measure of $\left[w_{1} \ldots w_{n}\right]$, and thus the frequency of the word $w_{1} \ldots w_{n}$, which should not be mistaken with its (symbolic) length.

If the transformation $T$ is minimal (i.e. every orbit is dense), all its trajectories have the same finite factors, and the language $L(T)$ is uniformly recurrent; the special words depend on the language and not on the individual trajectories; thus they are defined by any trajectory of $T$. If there is no periodic orbit, every word $w$ is a factor of a bispecial word; hence the bispecial words determine the finite factors of the trajectories, and thus the symbolic dynamical system $\left(X_{L(T)}, S\right)$.

1.3. Continued fractions. In Section 3 below, we shall use the generalized, or semi-regular continued fraction expansion

$$
\frac{1}{a_{1}+\varepsilon_{1} \frac{1}{a_{2}+\varepsilon_{2} \frac{1}{a_{3}+\cdots}}}
$$

for $a_{i}$ positive integers, $\varepsilon_{i}=-$ or,$+ \varepsilon_{i}=+$ if $a_{i}=1$. We denote the above expression by

$$
\left[0, a_{1} * \varepsilon_{1}, a_{2} * \varepsilon_{2}, \ldots\right] \text {. }
$$

We write $a_{n}$ with no sign if the expansion stops at $a_{n}$. In Section 2 we shall deal only with classic (Euclid) continued fraction expansions, and write only $a_{i}$ to denote $a_{i} *+$. The periodic sequence $a_{1} * \varepsilon_{1}, \ldots, a_{n} * \varepsilon_{n}, a_{1} * \varepsilon_{1}, \ldots, a_{n} * \varepsilon_{n}, \ldots$ is denoted by $\left(a_{1} * \varepsilon_{1}, \ldots, a_{n} * \varepsilon_{n}\right)^{\omega}$.

It is shown in [15] that $\left[0, a_{1} * \varepsilon_{1}, \ldots\right]$. has bounded partial quotients (for the Euclid algorithm) if and only if the $a_{i}$ are bounded and the number of consecutive $2 *-$ is bounded.

For a full study of the Lagrange spectrum, we refer the reader to the monograph [14]; the following definition is equivalent to the one given in the introduction of the present paper.

Definition 1.5. The Lagrange spectrum is the set of all finite values of

$$
\limsup _{k \rightarrow+\infty} \frac{1}{q_{k}\left|q_{k} \alpha-p_{k}\right|},
$$

for $\alpha$ irrational, the $\frac{p_{k}}{q_{k}}$ being the convergents of $\alpha$ for the Euclid algorithm.

Let us just recall that the Lagrange spectrum is a closed set, its lowest elements are $\sqrt{5}$, then $2 \sqrt{2}$, and discrete values (which, including these first two, are called the Lagrange numbers) up to a first accumulation point at 3 ; above 3 , its structure is more complicated and not yet fully known, but it contains every real number above a value (which is known to be optimal) near $4,52 \ldots$ 
Note that to get the Markov spectrum, we replace the upper limit by a supremum in the above definition; the Markov spectrum will not be used in the present paper.

1.4. Boshernitzan's $n e_{n}$. In [8] M. Boshernitzan introduced the following quantity:

Definition 1.6. Let $\left(X_{L}, S\right)$ be a minimal symbolic system. If $\mu$ is an $S$-invariant probability measure, for each natural integer $n$, we denote by $e_{n}(\mu)$ the smallest positive frequency of the words of length $n$ of $L$. If $\mu$ is the only invariant probability measure, $e_{n}(\mu)$ is simply denoted by $e_{n}$.

After partial results in [8] and [26], it was proved in [9] (see also the survey [18]) that whenever, for some invariant probability measure $\mu, n e_{n}(\mu)$ does not tend to 0 when $n$ tends to $+\infty$, then the system $\left(X_{L}, S\right)$ is uniquely ergodic. This sufficient condition for unique ergodicity has been known since [26] as Boshernitzan's citerion.

In the present paper, all systems considered are uniquely ergodic, and we consider the quantity $n e_{n}$ for its own sake. Thus we define

\section{Definition 1.7.}

$$
\mathcal{B}=\limsup _{n \rightarrow+\infty} \frac{1}{n e_{n}}, \quad \mathcal{B}^{\prime}=\liminf _{n \rightarrow+\infty} \frac{1}{n e_{n}} .
$$

Proposition 1.1. $\mathcal{B}$ and $\mathcal{B}^{\prime}$ are invariants of topological conjugacy among uniquely ergodic symbolic dynamical systems.

\section{Proof}

For such a conjugacy $\phi$ between two symbolic systems, the $i$-th coordinate of $\phi\left(\left(x_{n}\right)_{n \in \mathbb{N}}\right)$ depends only on $x_{i}, \ldots, x_{i+r}$ for a fixed integer $r$, see for example Lemma 5.1.14 of [24], and the image of the unique invariant probability measure on the first system is the one on the second system.

A first crude estimate can be given using the complexity function,

Lemma 1.2. $\mathcal{B} \geq \lim \sup _{n \rightarrow+\infty} \frac{p_{L}(n)}{n}, \mathcal{B}^{\prime} \geq \liminf _{n \rightarrow+\infty} \frac{p_{L}(n)}{n}$.

\section{Proof}

As the total measure of the space is 1 , we have $e_{n} \leq \frac{1}{p_{L}(n)}$.

This is enough to show that Boshernitzan's criterion is not a necessary condition: there are uniquely ergodic symbolic systems of exponential complexity [22], and thus with $n e_{n} \rightarrow 0$, see also the discussion at the end of Section 4. But of course the above lemma implies that the study of these invariants is interesting only for systems of sub-linear complexity, for which the question of necessity can be asked again.

In view of Theorem 2.5 below, we are led to define the following sets:

Definition 1.8. For a family of uniquely ergodic symbolic dynamical systems $\left(X_{a}, S\right), a \in \mathcal{F}$, the upper BL spectrum is the set of all values of $\mathcal{B}$ taken by the systems in this family, and the lower $\mathrm{BL}$ spectrum is the set of all values of $\mathcal{B}^{\prime}$ taken by the systems in this family. 


\section{Rotations AND the DyNAMicAl DEFinition of THE LAGRANGE SPECTRUM}

Surely there is nothing new to find about irrational rotations? The computation of $\mathcal{B}$ in Thorem 2.4 below, and the subsequent Theorem 2.5, which was the main motivation for the present paper, were known to P. Hubert and T. Monteil (private communications), but never written to our knowledge. The quantity $\frac{1}{\mathcal{B}^{\prime}}$ was indeed computed in [12] (see also [5]) as, for irrational rotations, it is equal to another invariant of topological conjugacy, the covering number by intervals [12], which involves covering the space by Rokhlin towers; the spectrum of its possible values is the object of a question in [12] and in [10], to which Theorem 2.6 below gives a first (to our knowledge), though belated and partial, answer.

Let $\alpha<\frac{1}{2}$ be an irrational number; the rotations with $\alpha>\frac{1}{2}$ are treated in a similar way and all the results in this section from Theorem 2.4 onwards remain valid; the rotation of angle $\alpha$, is also the two-interval exchange defined by

$$
T x= \begin{cases}x+\alpha & \text { if } x \in X_{1}=[0,1-\alpha[ \\ x-1+\alpha & \text { if } x \in X_{2}=[1-\alpha, 1[\end{cases}
$$

With this definition, a rotation admits a natural coding, by the partition of $X=[0,1[$ into $X_{1}$ and $X_{2}$. Then $L(T)$ has complexity $n+1$ and the trajectories are called Sturmian sequences. Irrational rotations are minimal and uniquely ergodic.

To get Theorem 2.4 below, we rely on a computation of both frequencies and lengths of factors of Sturmian sequences, which was done in [4], but which we provide again here by using a different version of the classic Euclid algorithm, making the computations quicker and ready to be generalized. This algorithm is the self-dual induction of [20] in the particular case of two intervals; all what we need to know is contained in the following proposition, which can also be proved directly without difficulty.

Proposition 2.1. If we build inductively real numbers $l_{n}$ and $r_{n}$ and words $w_{n}, M_{n}, P_{n}$ in the following way: $l_{1}=\alpha, r_{1}=1-2 \alpha, w_{1}=1, M_{1}=1, P_{1}=21$. Then

- whenever $l_{n}>r_{n}, l_{n+1}=l_{n}-r_{n}, r_{n+1}=r_{n}, w_{n+1}=w_{n} P_{n}, P_{n+1}=P_{n}, M_{n+1}=M_{n} P_{n}$;

- whenever $r_{n}>l_{n}, l_{n+1}=l_{n}, r_{n+1}=r_{n}-l_{n}, w_{n+1}=w_{n} M_{n}, P_{n+1}=P_{n} M_{n}, M_{n+1}=$ $M_{n}$.

Then the $w_{n}$ are all the nonempty bispecial words of $L(T), w_{n+1}$ being the shortest bispecial word beginning with $w_{n}$. The cylinder $\left[w_{n}\right]$ is the interval $\left[\alpha-l_{n}, \alpha+r_{n}[\right.$; its left and right subintervals separated by $\alpha$ are respectively $T\left[2 w_{n}\right]$ and $T\left[1 w_{n}\right]$, thus $l_{n}$, resp. $r_{n}$, is the frequency of the word $2 w_{n}$, resp. $1 w_{n}$. Also, $\left[w_{n} 1\right]=\left[w_{n} M_{n}\right]$ and $\left[w_{n} 2\right]=\left[w_{n} P_{n}\right]$.

The parameters $l_{n}$ and $r_{n}$ govern the process; the irrationality of $\alpha$ ensures that always $l_{n} \neq r_{n}$. Two fundamental relations come as direct consequences of the above formulas, as we check easily the considered quantities are not changed from $n$ to $n+1$ : for all $n$,

$$
\begin{gathered}
l_{n}\left|P_{n}\right|+r_{n}\left|M_{n}\right|=1, \\
\left|M_{n}\right|+\left|P_{n}\right|-\left|w_{n}\right|=2 .
\end{gathered}
$$

We define now the multiplicative form of this algorithm: 
Corollary 2.2. We define $l_{0}=\alpha, r_{0}=1-\alpha, M_{0}=1, P_{0}=2$, wo being the empty word. Then we define $a_{1}, a_{2}, \ldots$ such that $r_{n}>l_{n}$ for $0 \leq n \leq a_{1}-1, r_{n}<l_{n}$ for $a_{1} \leq n \leq a_{1}+a_{2}-1$, and so on. Let $o_{k}=a_{1}+\ldots a_{k}, o_{0}=0$; let $\alpha_{k}=r_{o_{k}}$ and $q_{k}=\left|M_{o_{k}}\right|$ if $k$ is even, $\alpha_{k}=l_{o_{k}}$ and $q_{k}=\left|P_{o_{k}}\right|$ if $k$ is odd.

Then for all $k \geq 0$, if $q_{-1}=q_{0}=1$,

$$
\alpha_{k}=a_{k+1} \alpha_{k+1}+\alpha_{k+2}, \quad q_{k+1}=a_{k+1} q_{k}+q_{k-1} .
$$

If $k$ is even $\alpha_{k+1}=l_{o_{k}}$ and $q_{k-1}=\left|P_{o_{k}}\right|$, while if $k$ is odd $\alpha_{k+1}=r_{o_{k}}$ and $q_{k-1}=\left|M_{o_{k}}\right|$.

The Euclid continued fraction expansion of $\alpha$ is $\left[0, a_{1}+1, a_{2}, \ldots\right]$, and the $q_{k}, k \geq 0$, are the denominators of the convergents of $\alpha$.

\section{Proof}

The written formulas are straightforward consequences of Proposition 2.1. They imply that $\frac{\alpha_{k+1}}{\alpha_{k}}=\frac{1}{a_{k+1}+\frac{\alpha_{k+2}}{\alpha_{k+1}}}$ and thus $\frac{\alpha_{k+1}}{\alpha_{k}}$ has the continued fraction expansion $\left[0, a_{k+1}, a_{k+2}, \ldots\right]$. Going to $k=0, \frac{\alpha_{1}}{\alpha_{0}}=\frac{\alpha}{1-\alpha}$ has the continued fraction expansion $\left[0, a_{1}, a_{2}, \ldots\right]$, which implies the last assertions.

The relations (1) and (2) imply $\left|w_{o_{k}}\right|=q_{k}+q_{k-1}-2$ and

$$
q_{k} \alpha_{k}+q_{k-1} \alpha_{k+1}=1
$$

We can now compute the frequencies as in [4]:

Lemma 2.3. For $\left|w_{n}\right|+2 \leq s \leq\left|w_{n+1}\right|$, the words of length $s$ have three possible frequencies which are $l_{n+1}, r_{n+1}$, and $l_{n+1}+r_{n+1}$; for $s=\left|w_{n}\right|+1$, the words of length s have two possible frequencies which are $l_{n}$ and $r_{n}$.

\section{Proof}

As in [4], we build the Rauzy graphs. If $w$ is not right special, $w^{\prime}$ is not left special, and $w \rightarrow w^{\prime}$, then $w$ and $w^{\prime}$ have the same frequency.

In the case of Sturmian sequences, for each $s$, there is one right special word of length $s$ and there is one left special word $G_{s}$ of length $s$; there are at most three frequencies, which are for example those of $G_{s}, 1 G_{s}$ and $2 G_{s}$, and these are exactly $l_{m}+r_{m}, l_{m}$ and $r_{m}$ for the smallest $m$ such that $\left|w_{m}\right| \geq s$.

But we need to check whether each of these frequencies is indeed the frequency of some word. We take an $n$ such that $r_{n}>l_{n}$, and let $p$ be the length of $w_{n}$. The possible frequencies of words of length $p$ are $l_{n}, r_{n}, l_{n}+r_{n}$, each one is indeed the frequency of a word of length $p$, as we take respectively $2 w_{n}$ deprived of its last letter, $1 w_{n}$ deprived of its last letter, and $w_{n}$. Then the words of length $p+1$ have possible frequencies $l_{n+1}, r_{n+1}, l_{n+1}+r_{n+1}$, which are respectively $l_{n}, r_{n}-l_{n}, r_{n}$; each of the two old frequencies $l_{n}, r_{n}$ is the frequency of a word of length $p+1$, as we take respectively $2 w_{n}$ and $1 w_{n}$, but the new one $r_{n}-l_{n}$ is not the frequency of any word of length $p+1$. The words of length $p+2$ have possible frequencies $l_{n+1}, r_{n+1}, l_{n+1}+r_{n+1}$, and each one is the frequency of a word of length $p+2$, as we take respectively $2 w_{n} 1,1 w_{n} 1$, and the prefix of length $p+2$ of $w_{n} M_{n}$; and this remains true (by extending the considered words to the right following $w_{n} M_{n}$ ) for $p+3, \ldots$ until we reach the length of $w_{n+1}$. And the reasoning is similar if $l_{n}>r_{n}$. 
Theorem 2.4. For a rotation of irrational angle $\alpha=\left[0, b_{1}, . ., . b_{n}, \ldots\right]$, if we define $v_{k}=$ $\left[0, b_{k}, b_{k-1}, \ldots b_{1}\right]$ and $t_{k}=\left[0, b_{k+1}, b_{k+2}, \ldots\right]$ then

$$
\mathcal{B}=\limsup _{k \rightarrow+\infty}\left(\frac{1}{v_{k}}+t_{k}\right)=\limsup _{k \rightarrow+\infty}\left(b_{k}+v_{k-1}+t_{k}\right), \quad \mathcal{B}^{\prime}=\liminf _{k \rightarrow+\infty}\left(1+t_{k} v_{k}\right) .
$$

\section{Proof}

Suppose $k$ is even. Then for $o_{k} \leq m \leq o_{k+1}-1, l_{m}=l_{o_{k}}$ and $l_{m}<r_{m}$; thus the smallest frequency of a word of length $n$ is $l_{o_{k}}$ for $\left|w_{o_{k}-1}\right|+2 \leq n \leq\left|w_{o_{k+1}-1}\right|+1$.

We know that $l_{o_{k}}=\alpha_{k+1}$ and $\left|w_{o_{k}}\right|=q_{k}+q_{k-1}-2$, and we check $\left|w_{o_{k}-1}\right|=q_{k}-2$. Thus the minimal value of $n e_{n}$ for $\left|w_{o_{k}-1}\right|+2 \leq n \leq\left|w_{o_{k+1}-1}\right|+1$ is $q_{k} \alpha_{k+1}$; the maximal value of $n e_{n}$ for $\left|w_{o_{k}-1}\right|+2 \leq n \leq\left|w_{o_{k+1}-1}\right|+1$ is $q_{k+1} \alpha_{k+1}$; all this is still true if $k$ is odd.

In Corollary 2.2, we identify $b_{1}$ with $a_{1}+1$ and $b_{i}$ with $a_{i}$ for $i \geq 2$. Thus we get $\frac{\alpha_{k+1}}{\alpha_{k}}=t_{k}$, while by construction of the $q_{k}$ we get $\frac{q_{k-1}}{q_{k}}=v_{k}$.

Then $\lim \sup _{n \rightarrow \infty} \frac{1}{n e_{n}}=\lim \sup _{k \rightarrow \infty} \frac{1}{q_{k} \alpha_{k+1}}=\lim \sup _{k \rightarrow \infty} \frac{q_{k+1} \alpha_{k+1}+q_{k} \alpha_{k+2}}{q_{k} \alpha_{k+1}}=$ $\lim \sup _{k \rightarrow \infty}\left(\frac{q_{k+1}}{q_{k}}+\frac{\alpha_{k+2}}{\alpha_{k+1}}\right)$, which is the first formula at stage $k+1$.

Similarly, $\lim \inf _{n \rightarrow \infty} \frac{1}{n e_{n}}=\liminf _{k \rightarrow \infty} \frac{1}{q_{k+1} \alpha_{k+1}}=\liminf _{k \rightarrow \infty} \frac{q_{k+1} \alpha_{k+1}+q_{k} \alpha_{k+2}}{q_{k+1} \alpha_{k+1}}=$ $\liminf _{k \rightarrow \infty}\left(1+\frac{\alpha_{k+2}}{\alpha_{k+1}} \frac{q_{k}}{q_{k+1}}\right)$, which is the second formula at stage $k+1$.

Theorem 2.5. The upper BL spectrum of the family of rotations is the union of the Lagrange spectrum and $+\infty$.

\section{Proof}

We check that $p_{k} \alpha_{k}+p_{k-1} \alpha_{k+1}=\alpha$ and $\left|p_{k} q_{k-1}-p_{k-1} q_{k}\right|=1$, thus $\liminf _{k \rightarrow \infty} q_{k}\left|q_{k} \alpha-p_{k}\right|$ is equal to $\lim \inf _{k \rightarrow \infty} q_{k} \alpha_{k+1}$.

As for the lower LB spectrum, it seems to have never been studied to our knowledge, and its study looks to be of the same level of difficulty as for the Lagrange spectrum. We give now some of the first results about it. Note that $\mathcal{B}^{\prime}$ is not the lower limit of $\frac{1}{q_{k}\left|q_{k} \alpha-p_{k}\right|}$ and thus is not directly linked to the quality of the approximation of $\alpha$ by rationals.

Theorem 2.6. The lower BL spectrum of the family of rotations has 1 as its smallest element, with $\mathcal{B}^{\prime}=1$ if and only if the angle has unbounded partial quotients. It is a closed set.

Its two largest elements are $\frac{5-\sqrt{5}}{2}=1,38196 \ldots$ and $3-\sqrt{3}=1,26794 \ldots$, and there is no other element above $\frac{5}{4}$.

It contains an accumulation point equal to $\sqrt{5}-1=1,2360 \ldots$

It contains the interval $\left[1,1+\frac{4}{83+18 \sqrt{2}}=1,03688 \ldots\right]$.

\section{Proof}

The first two assertions are straightforward consequences of the formula giving $\mathcal{B}^{\prime}$. The spectrum is closed by the standard reasoning of [14], Chapter 1, Corollary to Lemma 6, as every value greater than one is reached by an angle with bounded partial quotients, and for any sequence of angles such that the corresponding $\mathcal{B}^{\prime}$ converge to a number different from one, we can take the partial quotients uniformly bounded.

Let $\alpha=\left[0, b_{1}, . ., b_{n}, \ldots\right]$ be the angle of a rotation. 
Suppose that there are infinitely many $b_{k} \geq 4$; then for these $k, t_{k} v_{k} \leq \frac{1}{4}$.

If there are only 1,2 or 3 with infinitely many 3 , we have $t_{k} \leq\left[0,(13)^{\omega}\right]$. If $b_{k}=3$, we have $v_{k} \leq\left[0,3(31)^{\omega}\right]$ and we get $t_{k} v_{k} \leq \frac{\sqrt{21}-3}{\sqrt{21}+15}=0,2424 \ldots$

If there are only 1 and 2 with infinitely many 22 , if $b_{k}=b_{k+1}=2$ we get $t_{k} \leq\left[0,2(21)^{\omega}\right]$ and $v_{k} \leq\left[0,2(21)^{\omega}\right]$, thus $t_{k} v_{k} \leq \frac{12-6 \sqrt{3}}{9}=0,1786 \ldots$

If there are only 1 and 2 with no 22, infinitely many 2 and infinitely many 11 , if $b_{k}=2$, $b_{k+1}=b_{k+2}=1$ we get $v_{k} \leq\left[0,21(12)^{\omega}\right], t_{k} \leq\left[0,11(12)^{\omega}\right]$ and $t_{k} v_{k} \leq \frac{6-\sqrt{3}}{11} \frac{3-\sqrt{3}}{2}=0,2459 \ldots$

Thus the two highest values are reached for the angles $\left[0,(21)^{\omega}\right]$ and $\left[0,(1)^{\omega}\right]$, and the above estimates give a bound on the gap below them.

If we take $\alpha=\left[0,\left(1^{j} 2\right)^{\omega}\right]$, for a $j \geq 3$, the smallest value of $t_{n} v_{n}$ is reached when $b_{k}=2$ or $b_{k+1}=2$, and when $j$ tends to infinity, this value tends to $\left[0,(1)^{\omega}\right]\left[0,2(1)^{\omega}\right]=\sqrt{5}-2$. Thus we get an accumulation point, approached from above if $j$ is odd.

To prove the last assertion, we use Theorem 3.2 of [23]: any real number $s>1$ can be written $s=\left(r+\left[0, a_{1}, a_{2}, \ldots\right]\right)\left(r^{\prime}+\left[0, a_{1}^{\prime}, a_{2}^{\prime}, \ldots\right]\right)$ with all the $a_{i}$ and $a_{i}^{\prime}$ taking values $1,2,3$ or 4. Moreover, from the proof of this theorem we get ([23] p. 974) that if $s$ is at least $n^{2}+(\sqrt{2}-$ 1) $n+\frac{3-2 \sqrt{2}}{4}$ we can take both $r$ and $r^{\prime}$ greater or equal to $n$. Thus if $s$ is at least $\frac{83+18 \sqrt{2}}{4}$, we write $s$ in that way, with $r$ and $r^{\prime}$ at least 5 . We choose now a sequence $k_{n} \rightarrow+\infty$, and take the rotation of angle $\left[0, a_{k_{1}}, a_{k_{1}-1}, . ., a_{1}, r, r^{\prime}, a_{1}^{\prime}, \ldots a_{k_{1}}^{\prime}, a_{k_{2}}, a_{k_{2}-1}, . ., a_{1}, r, r^{\prime}, a_{1}^{\prime}, \ldots a_{k_{2}}^{\prime}, \ldots\right]$; then the minimal values of $t_{k} v_{k}$ are taken when $a_{k}=r$, and their lower limit is exactly $\frac{1}{s}$.

The third highest number in this spectrum is $\frac{16-4 \sqrt{6}}{5}=1,2404 \ldots$, as can be seen with longer computations; the point $\sqrt{5}-1$ is the highest accumulation point, but to prove it requires a machinery similar to the one used to prove Theorem 5 in Chapter 1 of [14].

\section{ThreE-INTERVAL EXCHANGES}

\subsection{The transformations.}

Definition 3.1. Given two numbers $0<\alpha, 0<\beta$ with $\alpha+\beta<1$, we define a three-interval exchange on $X=[0,1[$ by

$$
T x= \begin{cases}x+1-\alpha & \text { if } x \in X_{1}=[0, \alpha[ \\ x+1-2 \alpha-\beta & \text { if } x \in X_{2}=[\alpha, \alpha+\beta[ \\ x-\alpha-\beta & \text { if } x \in X_{3}=[\alpha+\beta, 1[.\end{cases}
$$

Throughout this section, we ask that $\alpha$ and $\beta$ satisfy the i.d.o.c condition of Keane, which means in that case that they do not satisfy any rational relation of the forms $p \alpha+q \beta=p-q$, $p \alpha+q \beta=p-q+1$, or $p \alpha+q \beta=p-q-1$, for $p$ and $q$ integers.

The points $\alpha$ and $\alpha+\beta$ are the discontinuities of $T$, while $\beta_{1}=1-\alpha-\beta$ and $\beta_{2}=1-\alpha$ are the discontinuities of $T^{-1}$. The i.d.o.c. condition ensures that the negative orbits of the discontinuities of $T$ are infinite and have an empty intersection (it is its original definition; see [16] for the equivalence with the one stated here). 
A three-interval exchange admits a natural coding, by the partition of $X$ into $X_{1}, X_{2}, X_{3}$. Under the i.d.o.c. condition, $(X, T)$ is minimal and uniquely ergodic and $L(T)$ has complexity $2 n+1$.

We recall that the induced, or first return, map $S$ of a transformation $T$ on a set $E$ is defined on $E$ by $S x=T^{g(x)} x$, where $g(x)$ is the smallest integer $r>0$ such that $T^{r} x$ is in $E$ (a finite $g(x)$ does indeed exist in all cases occurring in the present paper).

A three-interval exchange defined as above is always the induced map of the (irrational) rotation of angle $\frac{1-\alpha}{1+\beta}$ on the interval $\left[0, \frac{1}{1+\beta}[\right.$.

Throughout this section, we add the conditions $0<\alpha<\frac{1}{2}$, and $2 \alpha+\beta>1$; they ensure that the induction process described below does not have an irregular behaviour in the early stages: as is shown in [20], their absence modifies only a finite number of stages, and all the results in this section from Theorem 3.10 onwards remain valid without these extra conditions.

\subsection{The self-dual induction for 3 intervals. We state now some results from [20].}

Definition 3.2. We define an operation called self-dual induction, which builds numbers $l_{i, n}$ and $r_{i, n}, i=1,2$, through an infinite sequences of states in the following way:

State $I$ is defined by the relation $r_{1, n}=r_{2, n}$.

In Substate $I a l_{1, n}>r_{1, n}, l_{2, n}>r_{2, n}$. Then for $i=1,2$ we put $l_{i, n+1}=l_{i, n}-r_{i, n}, r_{i, n+1}=r_{i, n}$. For $n+1$ we are again in state $I$.

In Substate $I b l_{1, n}<r_{1, n}, l_{2, n}>r_{2, n}$. Then $l_{1 . n+1}=l_{1, n}, r_{1, n+1}=r_{1, n}, l_{2, n+1}=l_{2, n}-r_{2, n}$, $r_{2, n+1}=r_{2, n}$. For $n+1$ we are again in state $I$.

In Substate $I c l_{1, n}>r_{1, n}, l_{2, n}<r_{2, n}$. This is deduced from Ib by exchanging 1 and 2 , and for $n+1$ we are again in state $I$.

In Substate Id $l_{1, n}<r_{1, n}, l_{2, n}<r_{2, n}$. Then for $i=1,2, l_{i, n+1}=l_{i, n}, r_{i, n+1}=r_{i, n}-l_{i, n}$. For $n+1$ we are in state II described just below.

State $I I$ is defined by the relation $l_{1, n}+r_{1, n}=l_{2, n}+r_{2, n}$. Note that in this state $l_{1, n}>r_{2, n}$ if and only if $l_{2, n}>r_{1, n}$.

In Substate IIa $l_{1, n}>r_{2, n}, l_{2, n}>r_{1, n}$. Then for $i=1,2, l_{i . n+1}=l_{i, n}-r_{3-i, n}, r_{i, n+1}=r_{i, n}$. For $n+1$ we are in state III described below.

In Substate $I I b l_{1, n}<r_{2, n}, l_{2, n}<r_{1, n}$. Then for $i=1,2, r_{i . n+1}=r_{i, n}-l_{3-i, n}, l_{i, n+1}=l_{i, n}$. For $n+1$ we are in state $I$.

State $I I I$ is symmetrical to state $I$, with left and right exchanged, and the relation $l_{1, n}=$ $l_{2, n}$; there are four substates, III a to IIId, and the induction goes either to state III or to state II.

Proposition 3.1. For a given three-interval exchange, we build inductively real numbers $l_{i, n}$ and $r_{i, n}$ and words $w_{i, n}, M_{i, n}, P_{i, n}, i=1,2$, in the following way: $l_{1,1}=1-\alpha-\beta$, $r_{1,1}=2 \alpha+\beta-1=r_{2,1}, l_{2,1}=1-2 \alpha, w_{i, 1}=i$ and $M_{i, 1}=i$ for $i=1,2, P_{1,1}=31, P_{2,1}=2$.

$l_{i, n}$ and $r_{i, n}$ are built by Definition 3.2, starting from $n=1$, for which we are in State $I$.

For each $n>0$; let $s_{n}(1)=1, s_{n}(2)=2$ if for $n$ we are in $I$ or $I I I, s_{n}(1)=2, s_{n}(2)=1$ if for $n$ we are in $I I ; p_{n}(1)=1, p_{n}(2)=2$ if for $n$ we are in $I, p_{n}(1)=2, p_{n}(2)=1$ if for $n$ we are in $I I$ or $I I I ; m_{n}(1)=1, m_{n}(2)=2$ if for $n$ we are in $I I I, m_{n}(1)=2, m_{n}(2)=1$ if for $n$ we are in $I$ or $I I$. Then for $1 \leq i \leq 2$ 
- whenever $l_{i, n+1}<l_{i, n}$, then $w_{i, n+1}=w_{i, n} P_{s_{n} p_{n}(i), n}, P_{i, n+1}=P_{i, n}, M_{i, n+1}=M_{p_{n}(i), n} P_{i, n}$;

- whenever $r_{i, n+1}<r_{i, n}$, then $w_{i, n+1}=w_{i, n} M_{s_{n} m_{n}(i), n}, P_{i, n+1}=P_{m_{n}(i), n} M_{i, n}, M_{i, n+1}=$ $M_{i, n}$

- otherwise $w_{i, n+1}=w_{i, n}, P_{i, n+1}=P_{i, n}, M_{i, n+1}=M_{i, n}$.

Then the $w_{i, n}, n=1,2$, are all the nonempty bispecial words of $L(T)$; either $w_{i, n+1}=w_{i, n}$ or $w_{i, n+1}$ is the shortest bispecial word beginning with $w_{i, n}$. The cylinder $\left[w_{i, n}\right]$ is the interval $\left[\beta_{i}-l_{i, n}, \beta_{i}+r_{i, n}\left[;\right.\right.$ its left and right subintervals separated by $\beta_{i}$ are respectively $T\left[b w_{i, n}\right]$ and $T\left[a w_{i, n}\right]$ if $b w_{i, n}$ and $a w_{i, n}, a<b$, are the two left extensions of $w_{i, n}$; thus indeed $l_{1, n}$, $r_{1, n}, l_{2, n}, r_{2, n}$ are respectively the frequencies of the words $3 w_{1, n}, 2 w_{1, n}, 2 w_{2, n}, 1 w_{2, n}$. Also, $\left[w_{i, n} M_{s_{n} m_{n}(i), n}\right]=\left[w_{i, n} c\right]$ and $\left[w_{i, n} P_{s_{n} p_{n}(i), n}\right]=\left[w_{i, n} d\right]$, if $w_{i, n} c$ and $w_{i, n} d, d>c$, are the two right extensions of $w_{i, n}$.

A three-interval exchange defines an infinite sequence of states labelled Ia to Id, IIa, IIb, III a to IIId, following the rules of Definition 3.2, and such that each one of the four parameters $l_{1, n}, r_{1, n}, l_{2, n}, r_{2, n}$ is modified infinitely often. Conversely, every such sequence of states defines a three-interval exchange which generates it as described above.

Note that the i.d.o.c. condition ensures that we dont have $l_{1, n}=r_{1, n}$ or similar equalities. We check from the formulas that the following bilinear form is invariant by the induction, and thus for all $n$

$$
l_{1, n}\left|P_{1, n}\right|+r_{1, n}\left|M_{1, n}\right|+l_{2, n}\left|P_{2, n}\right|+r_{2, n}\left|M_{2, n}\right|=1 .
$$

The other relations are more complicated than in the case of rotations, we state them now but they are a straightforward consequence of Corollary 3.2 below:

- whenever for $n$ we are in $I I$,

$$
\left|P_{2, n}\right|+\left|M_{1, n}\right|=\left|M_{2, n}\right|+\left|P_{1, n}\right|+1=\left|w_{2, n}\right|+2=\left|w_{1, n}\right|+2 ;
$$

- whenever for $n$ we are in $I$,

$$
\left|P_{1, n}\right|+\left|M_{1, n}\right|=\left|w_{1, n}\right|+2, \quad\left|P_{2, n}\right|+\left|M_{2, n}\right|=\left|w_{2, n}\right|+1, \quad\left|P_{1, n}\right|=\left|P_{2, n}\right|+1 ;
$$

- whenever for $n$ we are in $I I I$,

$$
\left|P_{1, n}\right|+\left|M_{1, n}\right|=\left|w_{1, n}\right|+1, \quad\left|P_{2, n}\right|+\left|M_{2, n}\right|=\left|w_{2, n}\right|+2, \quad\left|M_{2, n}\right|=\left|M_{1, n}\right|+1 .
$$

We use now the self-dual induction to retrieve the frequencies and the lengths of the bispecial words, and this involves a multiplicative form of the algorithm; this has been done in [15] [16] [17] but in a form which is more complicated and less explicit (the frequencies of words are somewhat hidden) than the one which we deduce now from [20]; the results in this section are new, but could be deduced from [15] and [16], see [19] for the equivalence between the two forms of the algorithm. Thus the present paper is independent of all the previous ones except [20], and all the information we need from the latter are in Definition 3.2 and Proposition 3.1 above.

Corollary 3.2. For a given 3-iet, we define $o_{k}, k=1,2 \ldots$ to be the sequence of $n \geq 1$ such that for $n$ we are in state II; we define also $o_{0}=0$. Then for $k \geq 1$, we define two positive integers, $n_{k}$, resp. $m_{k}$, as the number of $o_{k-1}<n<o_{k}$ such that $w_{1, n+1} \neq w_{1, n}$, resp. $w_{2, n+1} \neq w_{2, n}$. Finally we define a sequence $\eta_{k}$, which is - , resp. + , whenever for $n=o_{k}-1$ we are in state $I$, resp. III. 
We define also parameters for $n=0$, namely, we are in state $I I, l_{1,0}=1-\alpha-\beta, r_{1,0}=\beta$, $l_{2,0}=1-2 \alpha, r_{2,0}=\alpha, w_{1,0}, w_{2,0}$ and $P_{1,0}$ are the empty word, $P_{2,0}=3, M_{1,0}=1, M_{2,0}=2$, $\eta_{0}=+$.

Then $o_{0}=0, \eta_{1}=-$. In the case $\eta_{k+1}=-$, for $0 \leq a \leq n_{k+1}-1,0 \leq b \leq m_{k+1}-1$,

$$
\begin{gathered}
r_{1, o_{k}+1+a}=r_{1, o_{k}}-l_{2, o_{k}}=r_{2, o_{k}}-l_{1, o_{k}}, \quad l_{1, o_{k}+1+a}=l_{1, o_{k}}-a\left(\left(r_{1, o_{k}}-l_{2, o_{k}}\right),\right. \\
r_{2, o_{k}+1+b}=r_{1, o_{k}}-l_{2, o_{k}}=r_{2, o_{k}}-l_{1, o_{k}}, \quad l_{2, o_{k}+1+b}=l_{2, o_{k}}-b\left(r_{1, o_{k}}-l_{2, o_{k}}\right), \\
w_{1, o_{k}+1+a}=w_{1, o_{k}} M_{1, o_{k}}\left(P_{2, o_{k}} M_{1, o_{k}}\right)^{a} \\
w_{2, o_{k}+1+b}=w_{2, o_{k}} M_{2, o_{k}}\left(P_{1, o_{k}} M_{2, o_{k}}\right)^{b} \\
M_{1, o_{k}+1+a}=M_{1, o_{k}}\left(P_{2, o_{k}} M_{1, o_{k}}\right)^{a}, \quad P_{1, o_{k}+1+a}=P_{2, o_{k}} M_{1, o_{k}} \\
M_{2, o_{k}+1+b}=M_{2, o_{k}}\left(P_{1, o_{k}} M_{2, o_{k}}\right)^{b}, \quad P_{2, o_{k}+1+b}=P_{1, o_{k}} M_{2, o_{k}} .
\end{gathered}
$$

Then $o_{k+1}=\max \left\{o_{k}+1+n_{k+1}, o_{k}+1+m_{k+1}\right\}$; between $\min \left\{o_{k}+n_{k+1}, o_{k}+m_{k+1}\right\}$ and $\max \left\{o_{k}+n_{k+1}, o_{k}+m_{k+1}\right\}$ one of the intervals is not modified, and

$$
\begin{gathered}
r_{1, o_{k+1}}=n_{k+1}\left(r_{1, o_{k}}-l_{2, o_{k}}\right)-l_{1, o_{k}}, \quad l_{1, o_{k+1}}=l_{1, o_{k}}-\left(n_{k+1}-1\right)\left(\left(r_{1, o_{k}}-l_{2, o_{k}}\right),\right. \\
r_{2, o_{k+1}}=m_{k+1}\left(r_{1, o_{k}}-l_{2, o_{k}}\right)-l_{2, o_{k}}, \quad l_{2, o_{k+1}}=l_{2, o_{k}}-\left(m_{k+1}-1\right)\left(\left(r_{1, o_{k}}-l_{2, o_{k}}\right),\right. \\
w_{1, o_{k+1}}=w_{1, o_{k}} M_{1, o_{k}}\left(P_{2, o_{k}} M_{1, o_{k}}\right)^{n_{k+1}-1} M_{2, o_{k}}\left(P_{1, o_{k}} M_{2, o_{k}}\right)^{m_{k+1}-1}, \\
w_{2, o_{k+1}}=w_{2, o_{k}} M_{2, o_{k}}\left(P_{1, o_{k}} M_{2, o_{k}}\right)^{m_{k+1}-1} M_{1, o_{k}}\left(P_{2, o_{k}} M_{1, o_{k}}\right)^{n_{k+1}-1}, \\
P_{1, o_{k+1}}=P_{1, o_{k}} M_{2, o_{k}} M_{1, o_{k}}\left(P_{2, o_{k}} M_{1, o_{k}}\right)^{n_{k+1}-1}, \quad M_{1, o_{k+1}}=M_{1, o_{k}}\left(P_{2, o_{k}, 1} M_{1, o_{k}}\right)^{n_{k+1}-1}, \\
P_{2, o_{k+1}}=P_{2, o_{k}} M_{1, o_{k}} M_{2, o_{k}}\left(P_{1, o_{k}} M_{2, o_{k}}\right)^{m_{k+1}-1}, \quad M_{2, o_{k+1}}=M_{2, o_{k}}\left(P_{1, o_{k}} M_{2, o_{k}}\right)^{m_{k+1}-1} .
\end{gathered}
$$

We get the case $\eta_{k+1}=+$ by exchanging the $l$ and $r$, or the $M$ and $P$, in both sides of all the above equalities.

A three-interval exchange defines an infinite sequence $\left(n_{k}, m_{k}, \eta_{k}\right), k \geq 1, n_{k} \geq 1, m_{k} \geq 1$, $\eta_{k}= \pm$, such that we do not have ultimately $n_{k}=1$ and $\eta_{k}$ constant, or $m_{k}=1$ and $\eta_{k}$ constant. Conversely, every such sequence defines a three-interval exchange which generates it as described above.

\section{Proof}

Through all the substates of state $I$ we have $r_{i, n+1}=r_{i, n}$, and the $l_{i, n}$ are decreased by a fixed quantity, so after a finite number of steps we are in $I d$ and proceed to $I I$, and similarly throughout stage $I I I$. Thus we are infinitely many times in state $I I$ and we can define $o_{k}$; $n_{k}$ and $m_{k}$ are at least one as for $n=o_{k}+1$ we are in state $I$ or $I I I$; we check that our parameters for $n=0$ are compatible with the ones already defined for $n=1$. Then we apply recursively Definition 3.2 and Proposition 3.1.

In the sequel, for a given three-interval exchange $T$, we shall use either the sequence $\left(n_{k}, m_{k}, \eta_{k}\right)$ or $\left(n_{k}, m_{k},-\eta_{k} \eta_{k+1}\right)$, which we both call the expansion of $T$; an expansion is admissible if we do not have ultimately $n_{k}=1$ and $\eta_{k}$ constant, or $m_{k}=1$ and $\eta_{k}$ constant. Note that [15] [16] [17] [19] use the expansion $\left(n_{k}, m_{k}, \epsilon_{k+1}\right)$ where $\epsilon_{k+1}=\eta_{k} \eta_{k+1}$.

We make now some further computations on the formulas above. 
Lemma 3.3. For all $k \geq 1$,

$$
\left(\begin{array}{c}
r_{1, o_{k-1}}+r_{2, o_{k-1}} \\
r_{1, o_{k-1}}-r_{2, o_{k-1}} \\
l_{1, o_{k-1}}+r_{1, o_{k-1}}
\end{array}\right)=\left(\begin{array}{ccc}
\eta_{k} & 0 & m_{k}+n_{k}-2 \eta_{k} \\
0 & 1 & \left(n_{k}-m_{k}\right) \eta_{k} \\
\eta_{k} & 0 & m_{k}+n_{k}-\eta_{k}
\end{array}\right)\left(\begin{array}{c}
r_{1, o_{k}}+r_{2, o_{k}} \\
r_{1, o_{k}}-r_{2, o_{k}} \\
l_{1, o_{k}}+r_{1, o_{k}}
\end{array}\right)
$$

Let $\Delta_{k}=l_{1, o_{k}}+r_{1, o_{k}}=l_{2, o_{k}}+r_{2, o_{k}}, t_{k}=\frac{\Delta_{k+1}}{\Delta_{k}}$. Then

$$
\begin{gathered}
r_{1, o_{k}}+r_{2, o_{k}}-\Delta_{k}=-\eta_{k+1} \Delta_{k+1}, \\
t_{k}=\left[0,\left(m_{k+1}+n_{k+1}\right) *-\eta_{k+1} \eta_{k+2},\left(m_{k+2}+n_{k+2}\right) *-\eta_{k+2} \eta_{k+3}, \ldots\right] \\
\frac{1-\alpha}{1+\beta}=\left[0,2 *+,\left(m_{1}+n_{1}\right) *-\eta_{1} \eta_{2},\left(m_{2}+n_{2}\right) *-\eta_{2} \eta_{3}, \ldots\right]
\end{gathered}
$$

\section{Proof}

(5) comes directly from the formulas in Corollary 3.2. These imply also (6), and $t_{k}=$ $-\eta_{k+1} \frac{r_{1, o_{k}}+r_{2, o_{k}}-\Delta_{k}}{\Delta_{k}}$, thus we get $t_{k}=\frac{1}{m_{k+1}+n_{k+1}-\frac{\eta_{k+1} \eta_{k+2}}{t_{k+1}}}$, and $t_{k}$ is given by the semi-regular continued fraction expansion (7). Going to $k=0$ we get a semi-regular continued fraction expansion of $\frac{\alpha+\beta}{1-\alpha}$, which can be put in the form (8).

Lemma 3.4. For all $k \geq 1$,

$$
\left(\begin{array}{c}
\left|P_{2, o_{k}}\right|+\left|P_{1, o_{k}}\right| \\
\left|P_{1, o_{k}}\right|-\left|P_{2, o_{k}}\right| \\
\left|M_{2, o_{k}}\right|+\left|P_{1, o_{k}}\right|
\end{array}\right)=V_{k}+\left(\begin{array}{ccc}
\eta_{k} & 0 & m_{k}+n_{k}-2 \eta_{k} \\
0 & 1 & n_{k}-m_{k} \\
\eta_{k} & 0 & m_{k}+n_{k}-\eta_{k}
\end{array}\right)\left(\begin{array}{c}
\left|P_{2, o_{k-1}}\right|+\left|P_{1, o_{k-1}}\right| \\
\left|P_{1, o_{k-1}}\right|-\left|P_{2, o_{k-1}}\right| \\
\left|M_{2, o_{k-1}}\right|+\left|P_{1, o_{k-1}}\right|
\end{array}\right) .
$$

where

$$
V_{k}=\left(\begin{array}{c}
n_{k}+1 \\
n_{k}-1 \\
n_{k}
\end{array}\right) \quad \text { if } \eta_{k}=-, \quad V_{k}=\left(\begin{array}{c}
m_{k}-1 \\
1-m_{k} \\
m_{k}
\end{array}\right) \quad \text { if } \eta_{k}=+.
$$

We define $z_{k}=\left|M_{2, o_{k}}\right|+\left|P_{1, o_{k}}\right|, v_{k}=\frac{q_{k-1}}{q_{k}}$, where

$$
q_{k+1}=\left(m_{k+1}+n_{k+1}\right) q_{k}-\eta_{k} \eta_{k+1} q_{k-1},
$$

$q_{-1}=0, q_{0}=1$. Then

$$
\begin{gathered}
\left|M_{2, o_{k}}\right|+\left|M_{1, o_{k}}\right|=z_{k}+\eta_{k} z_{k-1}+1+\eta_{k} . \\
\lim _{k \rightarrow+\infty}\left(z_{k} \Delta_{k}-\eta_{k} \eta_{k+1} z_{k-1} \Delta_{k+1}\right)=1 .
\end{gathered}
$$

$$
\lim _{k \rightarrow+\infty}\left(\frac{z_{k-1}}{z_{k}}-v_{k}\right)=0
$$

\section{Proof}

(9) comes directly from the formulas in Corollary 3.2. This implies in turn $\left|P_{2, o_{k}}\right|+\left|P_{1, o_{k}}\right|=$ $z_{k}-\eta_{k} z_{k-1}-\eta_{k}$ and thus (10).

We write now the relation (3) for $n=o_{k}+1$. Suppose $\eta_{k+1}=-1$. We notice that $r_{1, o_{k}+1}=r_{2, o_{k}+1}=r_{1, o_{k}}-l_{2, o_{k}}=r_{2, o_{k}}-l_{1, o_{k}}$ is also equal to $\Delta_{k+1}$, and thus $\Delta_{k+1}\left(\left|M_{2, o_{k}}\right|+\right.$ 
$\left.\left|M_{1, o_{k}}\right|\right)+l_{1, o_{k}}\left(\left|P_{2, o_{k}}\right|+\left|M_{1, o_{k}}\right|\right)+l_{2, o_{k}}\left(\left|M_{2, o_{k}}\right|+\left|P_{1, o_{k}}\right|\right)=1$. We recall that $\left|M_{2, o_{k}}\right|+\left|P_{1, o_{k}}\right|=z_{k}$ and $\left|P_{2, o_{k}}\right|+\left|M_{1, o_{k}}\right|=z_{k}+1$, while $\left|M_{2, o_{k}}\right|+\left|M_{1, o_{k}}\right|$ is given by (10). Because of (6), we get finally that $z_{k} \Delta_{k}+\eta_{k} z_{k-1} \Delta_{k+1}+\left(1+\eta_{k}\right) \Delta_{k+1}+l_{1, o_{k}}=1$. Similar computations when $\eta_{k+1}=+1$ give $z_{k} \Delta_{k}-\eta_{k} z_{k-1} \Delta_{k+1}-\eta_{k} \Delta_{k+1}+r_{2, o_{k}}=1$. In both cases we get (11).

(12) is a straightforward consequence of the definition of $q_{k}$.

We show now that $\frac{z_{k-1}}{z_{k}}-\frac{q_{k-1}}{q_{k}}$ tends to 0 when $k$ tends to $+\infty$. Indeed, from the matrix equality with $B_{k}$ and (10) we get $z_{k+1}=\left(m_{k+1}+n_{k+1}\right) z_{k}-\eta_{k} \eta_{k+1} z_{k-1}-\eta_{k} \eta_{k+1}+\theta_{k+1}$, where $\theta_{k}=n_{k}$ if $\eta_{k}=-1, \theta_{k}=m_{k}$ if $\eta_{k}=+1$. If $\Phi_{k}=q_{k} z_{k-1}-q_{k_{1}} z_{k}$, then $\Phi_{k+1}=$ $-\eta_{k} \eta_{k+1} \Phi_{k}-q_{k}\left(\theta_{k+1}+\eta_{k} \eta_{k+1}\right)$; we see that $\Phi_{k}$ grows more slowly than $q_{k}$, and this implies (13) as $z_{k}$ tends to infinity with $k$.

Note that the matrices in Lemmas 3.3 and 3.4 are the same except for one entry, the self-duality of the induction works but not as perfectly as for the rotations.

Though this will not be used in the sequel, the $q_{k}$ are indeed present in the computation of the lengths of the intervals, as we check that the third line of the matrix $A_{1} \ldots A_{k}$ is made with the entries $\eta_{k} q_{k-1}, 0, q_{k}-\eta_{k} q_{k-1}$, and this implies that $q_{k} \Delta_{k}-\eta_{k} \eta_{k+1} q_{k-1} \Delta_{k+1}=1-\alpha$. Thus from (11), (13), and the last equality, we deduce that $\frac{q_{k}}{z_{k}} \rightarrow 1-\alpha$ when $k$ tends to infinity.

From now on, when we study quantities involving large $k$, we shall always discard higher order terms such as $\left|w_{1, o_{k}}\right|-z_{k}$ or $z_{k} \Delta_{k}-z_{k-1} \Delta_{k+1}-1$, and replace $\frac{z_{k-1}}{z_{k}}$ by $v_{k}$.

\subsection{General formulas for $\mathcal{B}$ and $\mathcal{B}^{\prime}$.}

Lemma 3.5. The frequencies of words of length $n$ take at most six different values, which are $l_{i, q(i, n)}, r_{i, q(i, n)}$, and $l_{i, q(i, n)}+r_{i, q(i, n)}, i=1,2$, where $w_{i, q(i, n)}$ is the shortest bispecial word (in the families built by the self-dual induction) of length at least $n$.

\section{Proof}

We check that if $w=w_{1} \ldots w_{r}$ is in $L(T)$, so is $\bar{w}=w_{r} \ldots w_{1}$, with the same frequency as $w$ : this is done by induction on the length of $w$, as the frequencies of the words of length $n$ are deduced from the frequencies of words of length $n-1$ and the expression of the words of length $n$, see for example [8] or Proposition 4 of [21].

Then we build the Rauzy graphs, as was done in [8] without naming them. Given $w$, there exists at least one (left or right) special word linked by arrows with $w$, otherwise there is a periodic orbit and this contradicts the i.d.o.c. condition; thus $w$ has the same frequency as $v$, where $v$ is a word with $n$ letters, and either $v$ is left special, or $v \rightarrow v^{\prime}$ where $v^{\prime}$ is left special, or $v$ is right special, or $v^{\prime} \rightarrow v$ where $v^{\prime}$ is right special; by looking also at $\bar{w}$, we can suppose $v$ is left special or $v \rightarrow v^{\prime}$ where $v^{\prime}$ is left special.

There are two left special words with $n$ letters, $v_{i, n}$ beginning with $i$; let $L_{i, n}$ be the frequency of $b v_{i, n}, R_{i, n}$ the frequency of $a v_{i, n}$, for the two left extensions of $v_{i, n}, b>a$. Then the frequency of $v_{i, n}$ is $L_{i, n}+R_{i, n}$, and the frequency of a $v$ such that $v \rightarrow v_{i, n}$ is either $L_{i, n}$ or $R_{i, n}$. Moreover, let $W_{i, n}$ be the shortest bispecial word having $v_{i, n}$ as a prefix, which is also the shortest bispecial word beginning with $i$ and with length at least $n$; then $W_{i, n}$ is $w_{i, q}$ for $q=q(i, n), L_{i, n}=l_{i, q}$ and $R_{i, n}=r_{i, q}$. 
Lemma 3.5 was proved in [20] and can also be deduced from [8], we reprove it for sake of completeness.

Lemma 3.6. Suppose $r_{i, n+1}<r_{i, n}$; then each of the frequencies $l_{i, n+1}$ and $l_{i, n+1}+r_{i, n+1}$ is the frequency of a word of length $s$ for every $\left|w_{i, n}\right|+1 \leq s \leq\left|w_{i, n+1}\right|$; the frequency $r_{i, n+1}$ is the frequency of a word of length $s$ for every $\left|w_{i,}\right|+2 \leq s \leq\left|w_{i, n+1}\right|$, but not for $s=\left|w_{i, n}\right|+1$. The same is true with $l$ and $r$ exchanged.

\section{Proof}

This is the same as the last part of the proof of Lemma 2.3, mutatis mutandis: in particular, while the word $w_{n}$ could be preceded by two letters $2>1$, and followed by two letters $1<2$, here the word $w_{i, n}$ can be preceded by two letters $b>a$, and followed by two letters $c<d$, and $1 w_{n} 2$ is replaced by $a w_{i, n} d$ and so on.

Proposition 3.7. Let $T$ be a three-interval exchange with expansion $\left(n_{k}, m_{k},-\eta_{k} \eta_{k+1}\right)$; let $t_{k}$ be as in Lemma 3.3, $v_{k}$ as in Lemma 3.4; let

$$
\begin{gathered}
t_{k, l}=\frac{l_{2, o_{k}}}{l_{1, o_{k}}} \quad t_{k, r}=\frac{r_{2, o_{k}}}{r_{1, o_{k}}}, \quad v_{k, M}=\frac{\left(m_{k}-1\right) z_{k-1}+\left|M_{2, o_{k-1}}\right|}{\left(n_{k}-1\right) z_{k-1}+\left|M_{1, o_{k-1}}\right|}, \quad v_{k, M}^{\prime} \frac{m_{k} z_{k-1}+\left|M_{2, o_{k-1}}\right|}{n_{k} z_{k-1}+\left|M_{1, o_{k-1}}\right|}, \\
B_{k}=\frac{1}{1-\eta_{k} \eta_{k+1} t_{k}}+\frac{v_{k}}{1-v_{k}}, \quad B_{k}^{\prime}=\frac{1}{1+\eta_{k} \eta_{k+1} t_{k}}-\frac{v_{k}}{v_{k}+1} .
\end{gathered}
$$

Then $\mathcal{B}$ is the largest of the upper limits, when $k \rightarrow \infty$, of the following quantities

$$
\begin{gathered}
\frac{1}{v_{k}}-\eta_{k} \eta_{k+1} t_{k}, \\
\left(1+t_{k, l}\right)\left(1+v_{k, M}\right) B_{k}, \\
\left(1+\frac{1}{t_{k, l}}\right)\left(1+\frac{1}{v_{k, M}}\right) B_{k}, \\
\left(1+t_{k, r}\right)\left(1+v_{k, M}^{\prime}\right) B_{k}^{\prime}, \\
\left(1+\frac{1}{t_{k, r}}\right)\left(1+\frac{1}{v_{k, M}^{\prime}}\right) B_{k}^{\prime},
\end{gathered}
$$

where in formulas (15) to (18), when $\eta_{k}=+, t_{k, l}$ and $t_{k, r}$ have to be exchanged, and $v_{k, M}$ and $v_{k, M}^{\prime}$ have to be replaced by $v_{k, P}$ and $v_{k, P}^{\prime}$, where the $M$ are replaced by $P$.

\section{Proof}

We look at the possible frequencies of words from $n=o_{k-1}+1$ to $n=o_{k}$; suppose $\eta_{k}=-1$. Then $r_{1, n}$ is $\Delta_{k}$ from $n=o_{k-1}+1$ to $n=o_{k-1}+n_{k}$, then $r_{1, n}$ is $r_{1, o_{k}}$ from $n=o_{k-1}+n_{k}+1$ to $n=o_{k} ; r_{2, n}$ is $\Delta_{k}$ from $n=o_{k-1}+1$ to $n=o_{k-1}+m_{k}$, then $r_{2, n}$ is $r_{2, o_{k}}$ from $n=o_{k-1}+m_{k}$ to $n=o_{k} ; l_{1, n}$ takes values which are (by definition of the induction) larger than $r_{1, n}$ from $n=o_{k-1}+1$ to $n=o_{k-1}+n_{k}-1$, then $l_{1, n}$ is $l_{1, o_{k}}$ from $n=o_{k-1}+n_{k}$ to $n=o_{k} . l_{2, n}$ takes values which are (by definition of the induction) larger than $r_{2, n}$ from $n=o_{k-1}+1$ to $n=o_{k-1}+m_{k}-1$, then $l_{2, n}$ is $l_{2, o_{k}}$ from $n=o_{k-1}+m_{k}$ to $n=o_{k}$.

By Lemmas 3.5 and 3.6, we get that the smallest value of $n e_{n}$ between $n=\left|w_{1, o_{k-1}}\right|+2$ and $n=\left|w_{1, o_{k}}+1\right|$ is reached by one of five quantities: 
- $p r_{1, n}=p r_{2, n}$ for $p=\left|w_{1, o_{k-1}}\right|+2, n=o_{k-1}+1$,

- $p l_{1, n}$ for $p=\left|w_{1, o_{k-1}+n_{k}-1}\right|+2, n=o_{k}$,

- $p l_{2, n}$ for $p=\left|w_{2, o_{k-1}+m_{k}-1}\right|+2, n=o_{k}$,

- $p r_{1, n}$ for $p=\left|w_{1, o_{k-1}+n_{k}}\right|+2, n=o_{k}$,

- $p r_{2, n}$ for $p=\left|w_{2, o_{k-1}+m_{k}}\right|+2, n=o_{k}$.

Note that if $n_{k}=1$, the first quantity is smaller than the second, and could be dispensed with, but it is easier to use all the five quantities than to make many special subcases.

The first one is $z_{k-1} \Delta_{k}$, which gives formula (14) in view of (11).

The other ones are $l_{1, o_{k}}\left|w_{1, o_{k-1}+n_{k}-1}\right|, l_{2, o_{k}}\left|w_{2, o_{k-1}+m_{k}-1}\right|, r_{1, o_{k}}\left|w_{1, o_{k-1}+n_{k}}\right|, r_{2, o_{k}}\left|w_{2, o_{k-1}+m_{k}}\right|$.

We deal first with the third and fourth ones: we write (3) for $n=o_{k}-1$; we get that $\left(l_{1, o_{k}}+l_{2, o_{k}}\right) z_{k-1}+\Delta_{k}\left(\left|w_{1, o_{k-1}+n_{k}}\right|+\left|w_{2, o_{k-1}+n_{k}}\right|-2 z_{k-1}\right)$, which is equal to $\Delta_{k}\left(\left|w_{1, o_{k-1}+n_{k}}\right|+\right.$ $\left.\left|w_{2, o_{k-1}+m_{k}}\right|\right)-\left(r_{1, o_{k}}+r_{2, o_{k}}\right) z_{k-1}$, is close to 1 ; we write

$r_{1, o_{k}}\left|w_{1, o_{k-1}+n_{k}}\right|=\left(r_{1, o_{k}}+r_{2, o_{k}}\right)\left(\left|w_{1, o_{k-1}+n_{k}}\right|+\left|w_{2, o_{k-1}+m_{k}}\right|\right) \frac{r_{1, o_{k}}+r_{2, o_{k}}}{r_{1, o_{k}}} \frac{\left|w_{1, o_{k-1}+n_{k}}\right|+\left|w_{2, o_{k-1}+m_{k}}\right|}{\left|w_{1, o_{k-1}+n_{k}}\right|}$,

and notice that $\left|w_{1, o_{k-1}+n_{k}}\right|+\left|w_{2, o_{k-1}+m_{k}}\right|$ is close to $z_{k}+z_{k-1}$; after deducing $\frac{\Delta_{k}}{r_{1, o_{k}}+r_{2, o_{k}}}$ from (6) and imputting the values of the lengths of the bispecial words, this gives (17), and (18) is similar.

The expression (3) for $n=o_{k}-1$ is also close to $\left(l_{1, o_{k}}+l_{2, o_{k}}\right) z_{k-1}+\Delta_{k}\left(\left|w_{1, o_{k-1}+n_{k}-1}\right|+\right.$ $\left.\left|w_{2, o_{k-1}+m_{k}-1}\right|\right)$, which is thus close to 1 ; as $\left|w_{1, o_{k-1}+n_{k}-1}\right|+\left|w_{2, o_{k-1}+m_{k}-1}\right|$ is close to $z_{k}-z_{k-1}$, this gives (15) and (16).

And the case $\eta_{k}=1$ is deduced as usual.

To some extent, the quantities $B_{k}$ and $B_{k}^{\prime}$ measure the quality of approximation of the angle of the inducing rotation by the semi-regular continued fraction (8), which is best if $t_{k}$ or $v_{k}$ is close to 0 or 1 , see Proposition 5.1 of [15].

The value of $\mathcal{B}$ is not changed if we change a finite number of parameters of the expansion; this will be understated in the sequel when not recalled explicitely.

We remark that $v_{k}$ depends only on $m_{j}+n_{j}$ for $j \leq k$ and $-\eta_{j} \eta_{j+1}$ for $j \leq k-1 ; \eta_{k} \eta_{k+1} t_{k}$ depends only on $m_{j}+n_{j}$ for $j \geq k+1$ and $-\eta_{j} \eta_{j+1}$ for $j \geq k ; t_{k, l}$ and $t_{k, r}$ depend only on

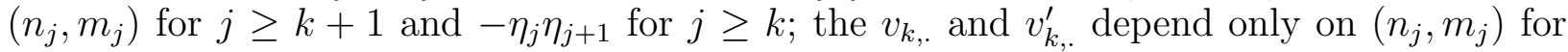
$j \leq k$ and $-\eta_{j} \eta_{j+1}$ for $j \leq k-1$.

Proposition 3.8. $\mathcal{B}^{\prime}$ is the smallest of the lower limits, when $k \rightarrow \infty$, of the following quantities

- when $\eta_{k}=-$ and $\left|w_{2, o_{k-1}+m_{k}-1}\right| \leq\left|w_{2, o_{k-1}+m_{k}}\right| \leq\left|w_{1, o_{k-1}+n_{k}-1}\right| \leq\left|w_{1, o_{k-1}+n_{k}}\right|$ : $\frac{1}{\Delta_{k}\left|w_{2, o_{k-1}+m_{k}-1}\right|}, \frac{1}{l_{2, o_{k}}\left|w_{2, o_{k-1}+m_{k} \mid}\right|}, \frac{1}{\min \left(l_{2, o_{k}}, r_{2, o_{k}}\right)\left|w_{1, o_{k-1}+n_{k}-1}\right|}, \frac{1}{\min \left(l_{1, o_{k}}, l_{2, o_{k}}, r_{2, o_{k}}\right)\left|w_{1, o_{k-1}+n_{k}}\right|}$, $\frac{1}{\min \left(l_{1, o_{k}}, l_{2, o_{k}}, r_{1, o_{k}}, r_{2, o_{k}}\right) z_{k}}$;

- when $\eta_{k}=-$ and $\left|w_{2, o_{k-1}+m_{k}-1}\right| \leq\left|w_{1, o_{k-1}+n_{k}-1}\right| \leq\left|w_{2, o_{k-1}+m_{k}}\right| \leq\left|w_{1, o_{k-1}+n_{k}}\right|$ : $\frac{1}{\Delta_{k}\left|w_{2, o_{k-1}+m_{k}-1 \mid}\right|}, \frac{1}{l_{2, o_{k}}\left|w_{1, o_{k-1}+n_{k}-1}\right|}, \frac{1}{\min \left(l_{1, o_{k}}, l_{2, o_{k}}\right)\left|w_{2, o_{k-1}+m_{k}}\right|}, \frac{1}{\min \left(l_{1, o_{k}}, l_{2, o_{k}}, r_{2, o_{k}}\right)\left|w_{1, o_{k-1}+n_{k}}\right|}$, $\frac{1}{\min \left(l_{1, o_{k}}, l_{2, o_{k}}, r_{1, o_{k}}, r_{2, o_{k}}\right) z_{k}}$;

- as in the first two cases with 1 and 2 exchanged, $m$ and $n$ exchanged;

- as in the first four cases with $r$ and $l$ exchanged and $\eta_{k}=+$. 


\section{Proof}

It is a straightforward consequence of the proof of Proposition 3.7.

We show how to compute effectively $\mathcal{B}$ for a particular family of cases:

Proposition 3.9. Let $n \geq m \geq 1$ be two integers; we consider a three-interval exchange such that for all $k n_{k}=n, m_{k}=m, \eta_{k} \eta_{k+1}=-1$. Then $t$ is the smaller root of the polynomial $X^{2}+(m+n) X-1$, and $\mathcal{B}$ is the largest of the three quantities

$$
\begin{gathered}
t+\frac{1}{t}, \quad\left(\frac{(m+n)^{2}}{m^{2}}\right)\left(\frac{1}{1+t}+\frac{t}{1-t}\right) \\
\left(\frac{2(1-t)^{2}}{(1-t)^{2}+(m-n) t}\right)\left(\frac{2(1+t)^{2}}{(1+t)^{2}+(n-m) t}\right)\left(\frac{1}{1+t}+\frac{t}{1-t}\right) .
\end{gathered}
$$

\section{Proof}

Then for every $k$ we have $t_{k}=v_{k}=t$. Thus $r_{1, o_{k}}+r_{2, o_{k}}=\Delta_{k}\left(1-\eta_{k+1}\right) t$, while $r_{1, o_{k}}-r_{2, o_{k}}=$ $\Delta_{k} \eta_{k+1} u$, for some constant $u$; by (5) we get $u=\frac{(n-m) t}{1+t}$. Similarly, up to higher order terms, $z_{k-1}=t z_{k}$, and $\left|M_{1, o_{k}}\right|-\left|M_{2, o_{k}}\right|=z_{k} u^{\prime}$ with $u^{\prime}=\frac{(n-m) t}{1-t}$.

Without loss of generality, we suppose $\eta_{k}=-1$, and compute the various quantities in formulas (14) to (18). Formula (14) becomes $t+\frac{1}{t}$, and $B_{k}=B_{k}^{\prime}=\frac{1}{1+t}+\frac{t}{1-t}$. Note also that formula (17) gives a smaller estimate than (18) and thus will not be used.

We look now at $1+\frac{1}{t_{k, r}}$; inputting the above values, we get that it is equal to $\frac{2-2 t^{2}}{1-t^{2}+(m-n) t}$; but, using the equation defining $t$, we check that it is just equal to $\frac{m+n}{m}$. Similarly, we get $1+t_{k, l}=\frac{2(1+t)^{2}}{(1+t)^{2}+(m-n) t}$ and $1+\frac{1}{t_{k, l}}=\frac{2(1+t)^{2}}{(1+t)^{2}+(n-m) t}$, and these do not seem to admit simpler expressions.

In the same way, we get that $1+\frac{1}{v_{k, M}^{\prime}}$ is equal to $\frac{2+\frac{2}{t}}{2 m+1+t+(m-n) \frac{t}{1-t}}$; this last quantity turns out to be also equal to $\frac{m+n}{m}$. Then $1+v_{k, M}$ is equal to $\frac{-2+\frac{2}{t}}{2 n-1+t+(n-m) \frac{t}{1-t}}$, which, by using the equation defining $t$, is also equal to $\frac{2(1-t)^{2}}{(1-t)^{2}+(n-m) t}$. Finally $1+\frac{1}{v_{k, M}}$ can be recovered from the last quantity and is equal to $\frac{2(1-t)^{2}}{(1-t)^{2}+(m-n) t}$. But at this stage we notice that formula (15) gives a smaller estimate than (16) and thus will not be used. Thus we have the three claimed formulas.

\subsection{The upper BL spectrum: smallest elements.}

Theorem 3.10. The smallest element in the upper BL spectrum of three-interval exchange transformations, and the only one below $\frac{12+29 \sqrt{3}}{13}=4,786 \ldots$, is $2 \sqrt{5}=4,47 \ldots$ It is reached if and only if for all $k$ large enough $m_{k}=n_{k}=2$ and $\eta_{k} \eta_{k+1}=-$.

The spectrum is a closed set and contains an accumulation point equal to 6 .

\section{Proof}

We look at formulas (14) to (18). The maximum of (15) and (16) is at least $4 B_{k}$, while the maximum of (17) and (18) is at least $4 B_{k}^{\prime}$.

We take a three-interval exchange with expansion $\left(n_{k}, m_{k}, \eta_{k}\right)$ and look at the sequence $\left(a_{k}, \varepsilon_{k}\right)$ where $a_{k}=m_{k}+n_{k}, \varepsilon_{k}=-\eta_{k} \eta_{k+1}$. 
If there are infinitely many $a_{k} \geq 6$, or $\left(a_{k}, \varepsilon_{k}\right)=(5,+)$, then for infinitely many $k$ formula (14) gives a bound greater or equal to 5 .

If there are only $a_{k} \leq 4$ or $\left(a_{k}, \varepsilon_{k}\right)=(5,-)$, and for infinitely many $k$ we have $\varepsilon_{k}=-;$ then we have always $t_{k} \geq\left[0,(5 *-)^{\omega}\right]=\frac{5-\sqrt{21}}{2}$ and also $v_{k} \geq\left[0,(5 *-)^{\omega}\right]$. Then if $\varepsilon_{k}=-, 4 B_{k}$ is at least $\frac{4 \sqrt{21}}{3}=6,11 \ldots$

If there are only $\left(a_{k}, \varepsilon_{k}\right)=(3,+),\left(a_{k}, \varepsilon_{k}\right)=(4,+)$ or $\left(a_{k}, \varepsilon_{k}\right)=(2,+)$, with infinitely many $(2,+)$; then we have always $v_{k} \geq\left[0,(2 *+, 4 *+)^{\omega}\right]=\sqrt{6}-2$. If $\left(a_{k+1}, e_{k+1}\right)=(2,+)$, then $t_{k} \geq\left[0,2 *+(2 *+, 4 *+)^{\omega}\right]=\frac{1}{\sqrt{6}}$ and thus $4 B_{k}^{\prime}$ is at least $\frac{8+8 \sqrt{6}}{5}=5,519 \ldots$.

If there are only $\left(a_{k}, \varepsilon_{k}\right)=(3,+)$ or $\left(a_{k}, \varepsilon_{k}\right)=(4,+)$, with infinitely many $(3,+)$; then we have always $v_{k} \geq\left[0,(3 *+, 4 *+)^{\omega}\right]=\frac{4 \sqrt{3}-6}{3}$, If $\left(a_{k+1}, e_{k+1}\right)=(3,+)$, then $t_{k} \geq[0,3 *+(3 *$ $\left.+, 4 *+)^{\omega}\right]=\frac{4 \sqrt{3}-3}{13}$ and thus $4 B_{k}^{\prime}$ is at least $\frac{12+29 \sqrt{3}}{13}=4,786 \ldots$

If there are only $\left(a_{k}, \varepsilon_{k}\right)=(4,+)$, with for infinitely many $k m_{k} \neq n_{k}$; then $t_{k}=v_{k}=\sqrt{5}-2$. We take a $k$ such that $m_{k} \neq n_{k}$; without loss of generality we take $\eta_{k}=-$. If $m_{k}=1$ and $n_{k}=3$, then $r_{1, o_{k}}>r_{2, o_{k}}$, and $\frac{1}{v_{k, M}^{\prime}}=\frac{3 z_{k-1}+\left|M_{1, o_{k-1}}\right|}{z_{k-1}+\left|M_{2, o_{k-1}}\right|} \geq \frac{3 z_{k-1}}{z_{k-1}+\left|M_{1, o_{k-1}}\right|+\left|M_{2, o_{k-1}}\right|}=\frac{3 z_{k-1}}{2 z_{k-1}+z_{k-2}}$; as $z_{k-2}=v_{k-1} z_{k-1}$, formula (18) gives at least $\frac{15+7 \sqrt{5}}{4}=7,663 \ldots$ If $m_{k}=3$ and $n_{k}=1$, formula (17) gives the same estimate.

There remains only the case $\left(a_{k}, \varepsilon_{k}\right)=(4,+)$ and $m_{k}=n_{k}$, where $\mathcal{B}$ has the claimed value; note that each of the formulas (14) to (18) gives the same result.

If we take $\left(a_{k}, \varepsilon_{k}\right)$ to be $\left((2,+)^{2},(4,+)^{j}\right)^{\omega}$, and $m_{k}=n_{k}$, the value of $\mathcal{B}$ is reached (among others) by $4 B_{k}^{\prime}$ for $a_{k}=2$ and $a_{k-1}=4$; when $j$ tends to infinity, these values of $v_{k}$ and $t_{k}$ both tend to $\left[0,2 *+(4 *+)^{\omega}\right]=\frac{1}{\sqrt{5}}$, and thus $\mathcal{B}$ tends to 6 . The spectrum is closed by the standard reasoning of [14], Chapter 1 , Corollary to Lemma 6 , as every finite value is reached with bounded $m_{k}$ and $n_{k}$, and for any sequence of three-interval exchanges such that the corresponding $\mathcal{B}$ converge to a finite number, we can take the $m_{k}$ and $n_{k}$ uniformly bounded (and $+\infty$ is in the spectrum, see Proposition 3.11 below). 6 is indeed an accumulation point, approached from below if $j$ is even.

The above computations illustrate how $\mathcal{B}$ is easiest to compute when $m_{k}=n_{k}$ for all $k$; when $m_{k} \neq n_{k}$, the situation becomes much more complicated; indeed, some of the bounds in the above computations may look quite crude, as for example if $m_{k}+n_{k}=3$ we cannot have $m_{k}=n_{k}$ and the bounds $4 B_{k}$ and $4 B_{k}^{\prime}$ are never reached, but in general they are not easy to improve.

In view of the considerations above, the values of $\mathcal{B}$ between $2 \sqrt{5}$ and 6 were found only by trial and error; the second value is very likely to be $4 \sqrt{2}=5,65 \ldots$, which is reached when $\left(n_{k}, m_{k},-\eta_{k} \eta_{k+1}\right)$ is either $(1,1,+)^{\omega}$ or $(3,3,-)^{\omega}$; the latter gives the minimal value for $m_{k}$ and $n_{k}$ constant and $\eta_{k} \eta_{k+1}=+1$. The third value we found is $\frac{20 \sqrt{26}}{17}=5,9988 \ldots$, which is reached for $\left((1,1,-)^{2},(2,2,-)^{2}\right)^{\omega}$ and the fourth one is $\frac{4 \sqrt{209306}}{305}=5,999996 \ldots$, which is reached for $\left((1,1,-)^{2},(2,2,-)^{4}\right)^{\omega}$.

Thus the first, second, third, fourth smallest element we found in the upper BL spectrum of three-interval exchange transformations is respectively twice the first, second, sixth and twelfth Lagrange number. Though of course we might have missed some values, it seems likely that the upper BL spectrum of three-interval exchanges below 6 is strictly included in 
twice the Lagrange spectrum below 3; thus we conjecture that 6 is the lowest accumulation point of our spectrum.

When $\left(n_{k}, m_{k},-\eta_{k} \eta_{k+1}\right)$ is $(2,2,+)^{\omega}$, the values of $\alpha=\beta$ and the angle of the inducing rotation can be deduced from (8); we get $\alpha=\beta=\frac{3-\sqrt{5}}{2}$, and the inducing rotation is of angle $\frac{1}{\sqrt{5}}$; as in that case (8) gives a classical continued fraction expansion, the $\mathcal{B}$ of the rotation is computed by Theorem 2.4, and we check that the $\mathcal{B}$ of the interval exchange is the one of the inducing rotation. By similar computations, for $(1,1,+)^{\omega}$, the $\mathcal{B}$ of the interval exchange is twice the one of the inducing rotation. For $\left((1,1,+)^{2},(2,2,+)^{j}\right)^{\omega}$ with $j$ large, the $\mathcal{B}$ of the interval exchange is close to 6 , while the one of the inducing rotation is between 4 and 5 .

A partial analogy with the Lagrange spectrum is that in the latter the sequence increasing to 3 is given by angles whose Euclid continued fraction expansion is $\left[0,\left(2^{2} 1^{2 j}\right)^{\omega}\right]$, while in our upper BL spectrum the sequence increasing to 6 is given by quantities $t_{k}$ whose Euclid continued fraction expansion is $\left[0,\left(2^{2} 4^{2 j}\right)^{\omega}\right]$; but it fails for the angles whose classic continued fraction expansion is $\left[0,\left(2^{4} 1^{2}\right)^{\omega}\right]$, which gives a rotation with $\mathcal{B}=2,9992 \ldots$, while the threeinterval exchange for which $\left(n_{k}, m_{k},-\eta_{k} \eta_{k+1}\right)$ is $\left((1,1,+)^{4},(2,2,+)^{2}\right)^{\omega}$ has $\mathcal{B}=6,06 \ldots$.

All the values of $\mathcal{B}$ we have found below 6 are reached by the estimates $4 B_{k}$ or $4 B_{k}^{\prime}$, though when $\left(n_{k}, m_{k},-\eta_{k} \eta_{k+1}\right)$ is either $(2,2,+)^{\omega}$ or $(3,3,-)^{\omega}$ they are also reached by formula (14). The smallest value we found without $m_{k}=n_{k}$ for all $k$ large enough is $\frac{5 \sqrt{29}}{4}=6,73 \ldots$, which is reached when $\left(n_{k}, m_{k},-\eta_{k} \eta_{k+1}\right)$ is $(3,2,+)^{\omega}$; for $(3,1,+)^{\omega}$ we get $\mathcal{B}=8 \sqrt{5}$.

\subsection{The upper BL spectrum: largest elements.}

Proposition 3.11. For a three-interval exchange, $\mathcal{B}=+\infty$ if and only if the angle of the inducing rotation has unbounded partial quotients for the Euclid algorithm.

\section{Proof}

By the formulas in Proposition 3.7, $\mathcal{B}=+\infty$ if and only if either $m_{k}+n_{k}$ is unbounded, or $t_{k}$ or $v_{k}$ takes values arbitrarily close to one. The last two possibilities are equivalent to the existence of unbounded strings of $m_{k}+n_{k}=2,-\eta_{k} \eta_{k+1}=-$, and this gives the result by (8) and Section 1.3.

The upper BL spectrum above 6 seems to become quite complicated, maybe more than the Lagrange spectrum above 3 . However, we can find enough values given by formula (14) (this happens when all $m_{k}=n_{k}$ and some of them are large enough) to fill an interval $[C,+\infty[$. This involves adapting to semi-regular continued fraction expansions the famous Theorem 3.1 of $[23]$ :

Lemma 3.12. Let $G$ be the set of numbers $\left[0, a_{1} * \varepsilon_{1}, \ldots, a_{n} * \varepsilon_{n}, \ldots ..\right]$ where all the $a_{i}$ are $2,4,6$ or 8 , the $\varepsilon_{i}$ are - or + , and there are never two consecutive $2 *-$; then $G+$ $G$ is the interval $\left[\frac{2 \sqrt{195}-24}{17}, \frac{2 \sqrt{195}-24}{3}\right]=[0,23108 \ldots, 1,30949 \ldots]$, and $G-G$ is the interval $\left[\frac{168-14 \sqrt{195}}{51}, \frac{14 \sqrt{195}-168}{51}\right]=[-0,53920 \ldots, 0,53920 \ldots]$.

\section{Proof}

We follow the proof of Theorem 2 in Chapter 4 of [14], which in turns follows [23]. The largest number in $G$ is $\left[0,(2 *-, 2 *+, 8 *+)^{\omega}\right]=\frac{\sqrt{195}-12}{3}$, and the smallest one is $[0,(8 *+, 2 *-, 2 *$ $\left.+)^{\omega}\right]=\frac{\sqrt{195}-12}{17}$. We can build $G$ as a Cantor set in the following way: we start form the 
interval $\left[\frac{\sqrt{195}-12}{17}, \frac{\sqrt{195}-12}{3}\right]$. Then, for each sequence $\left(b_{1}, \varepsilon_{1}\right), \ldots\left(b_{n}, \varepsilon_{n}\right)$ with no two consecutive $(2,-)$ and $b_{i}=2,4,6,8$ : if the number of $\varepsilon_{i}$ which are + is 0 or even, we delete the intervals ]$\left[0, b_{1} * \varepsilon_{1}, \ldots, b_{n} * \varepsilon_{n}, a *+,(8 *+, 2 *-, 2 *+)^{\omega}\right],\left[0, b_{1} * \varepsilon_{1}, \ldots, b_{n} * \varepsilon_{n}, a *-,(8 *+, 2 *-, 2 *+)^{\omega}\right][$ for $a=2,4,6,8$, and $]\left[0, b_{1} * \varepsilon_{1}, \ldots, b_{n} * \varepsilon_{n},(a+2) *-,(2 *-, 2 *+, 8 *+)^{\omega}\right],\left[0, b_{1} * \varepsilon_{1}, \ldots, b_{n} *\right.$ $\left.\varepsilon_{n}, a *+,(2 *-, 2 *+, 8 *+)^{\omega}\right]\left[\right.$ for $a=2,4,6$; if the number of $\varepsilon_{i}$ which are + is odd, we delete the intervals $]\left[0, b_{1} * \varepsilon_{1}, \ldots, b_{n} * \varepsilon_{n}, a *-,(8 *+, 2 *-, 2 *+)^{\omega}\right],\left[0, b_{1} * \varepsilon_{1}, \ldots, b_{n} * \varepsilon_{n}, a *+,(8 *\right.$ $\left.+, 2 *-, 2 *+)^{\omega}\right][$ for $a=2,4,6,8$, and $]\left[0, b_{1} * \varepsilon_{1}, \ldots, b_{n} * \varepsilon_{n}, a *+,(2 *-, 2 *+, 8 *+)^{\omega}\right],\left[0, b_{1} *\right.$ $\left.\varepsilon_{1}, \ldots, b_{n} * \varepsilon_{n},(a+2) *-,(2 *-, 2 *+, 8 *+)^{\omega}\right][$ for $a=2,4,6$. We check that this dissection can be done in successive stages, where each time we delete the interval $A_{2}$ from the interval $A_{1} \cup A_{2} \cup A_{3}$, and the length of $A_{2}$ is smaller than the length of $A_{1}$ and than the length of $A_{3}$; then Lemmas 2 to 4 of [14] give the result. The same reasoning works for $G+(-G)$.

Theorem 3.13. The upper BL spectrum of the family of three-interval exchanges contains the interval $[12+2 \sqrt{2}=14,828 \ldots,+\infty]$.

\section{Proof}

By Proposition 3.11, the upper BL spectrum contains $+\infty$. Let $s$ be a real number larger than $12+2 \sqrt{2}$; by Lemma 3.12, we can write $s=r+\varepsilon\left[0, a_{1} * \varepsilon_{1}, \ldots, a_{n} * \varepsilon_{n}, \ldots.\right]+\varepsilon^{\prime}\left[0, a_{1}^{\prime} *\right.$ $\left.\varepsilon_{1}^{\prime}, \ldots, a_{n}^{\prime} * \varepsilon_{n}^{\prime}, \ldots ..\right]$, where $r$ is an even positive integer, all the $a_{i}$ and $a_{i}^{\prime}$ are $2,4,6$ or 8 , the $\varepsilon_{i}$, $\varepsilon_{i}^{\prime}, \varepsilon$ and $\varepsilon^{\prime}$ are - or + , and there are never two consecutive $2 *-$ in the $a_{n} * \varepsilon_{n}$ and $a_{n}^{\prime} * \varepsilon_{n}^{\prime}$. We choose some increasing sequence $k_{n}$ such that $a_{k_{n}}^{\prime} * \varepsilon_{k_{n}}^{\prime}$ is not $2 *-$.

We define the expansion $\left(m_{k}+n_{k},-\eta_{k} \eta_{k+1}\right)$ to be $\left(a_{k_{1}}, \varepsilon_{k_{1}-1}\right), \ldots .\left(a_{3}, \varepsilon_{2}\right)\left(a_{2}, \varepsilon_{1}\right)\left(a_{1}, \varepsilon\right)\left(r, \varepsilon^{\prime}\right)$ $\left(a_{1}^{\prime}, \varepsilon_{1}^{\prime}\right) \ldots\left(a_{k_{1}}^{\prime}, \varepsilon_{k_{1}}^{\prime}\right)\left(a_{k_{2}}, \varepsilon_{k_{2}-1}\right), \ldots .\left(a_{3}, \varepsilon_{2}\right)\left(a_{2}, \varepsilon_{1}\right)\left(a_{1}, \varepsilon\right)\left(r, \varepsilon^{\prime}\right)\left(a_{1}^{\prime}, \varepsilon_{1}^{\prime}\right) \ldots\left(a_{k_{2}}^{\prime}, \varepsilon_{k_{1}}^{\prime}\right) \ldots$ We take now the three-interval exchange for which $m_{k}+n_{k}$ and $-\eta_{k} \eta_{k+1}$ are defined in that way, and $m_{k}=n_{k}$ for all $k$; it exists as this gives an admissible expansion. By Proposition $3.7 \mathcal{B}$ is the maximum of the upper limits of $\frac{1}{v_{k}}-\eta_{k} \eta_{k+1} t_{k}$, and of $4 B_{k}$ and $4 B_{k}^{\prime}$. As $r$ is at least 14 , the first of these upper limits can be taken on those $k$ for which $m_{k}+n_{k}=r$, and is exactly $s$.

We look now at the other two upper limits, for which we need an upper bound for $t_{k}$ and $v_{k}$. If we compute $t_{k}$ when $\left(m_{k}+n_{k},-\eta_{k} \eta_{k+1}\right)$ is inside a string of $\left(a_{n}^{\prime}, \varepsilon_{n}^{\prime}\right)$, we do not see two consecutive $(2,-)$ in that string, while, when we start inside a string of $\left(a_{n}, \varepsilon_{n}\right)$, in that string we do not see $(a,-)$ followed by $(2,-)$ followed by $(2, f)$ for any $a$ and $f$, and the two cases are exchanged if we compute $v_{k}$; in both cases starting from a $\left(r, \varepsilon^{\prime}\right)$ or near a junction of two different strings does not change the bounds. Thus either $t_{k}$ is at most $[0,2 *-, 2]=\frac{2}{3}$, and $v_{k}$ is at most $\left[0,2 *-,(2 *-, 4 *-)^{\omega}\right]=\frac{1}{\sqrt{2}}$, or $v_{k}$ is at most $[0,2 *-, 2]=\frac{2}{3}$, and $t_{k}$ is at most $\left[0,2 *-,(2 *-, 4 *-)^{\omega}\right]=\frac{1}{\sqrt{2}}$; these bounds can be improved when $\left(m_{k}+n_{k},-\eta_{k} \eta_{k+1}\right)=(2,-)$, then either $t_{k}$ is at most $[0,2]=\frac{1}{2}$, and $v_{k}$ is at most $\left[0,2 *-,(4 *-, 2 *-)^{\omega}\right]=2-\sqrt{2}$, or $v_{k}$ is at most $[0,2 *-, 2]=\frac{2}{3}$, and $t_{k}$ is at most $\left[0,(2 *-, 4 *-)^{\omega}\right]=2-\sqrt{2}$; also, when $m_{k}+n_{k}$ is at least $4, v_{k}$ is at most either $\left[0,4 *-,(2 *-, 4 *-)^{\omega}\right]=\frac{2-\sqrt{2}}{2}$, or $[0,4 *-, 2 *-, 2]=\frac{3}{10}$. We input these values in the quantities we want to bound, together with $t_{k}>0, v_{k}>0$; the worst case happens when $\left(m_{k}+n_{k},-\eta_{k} \eta_{k+1}\right)=(2,-)$, and we get that the last two upper limits are not larger than $12+2 \sqrt{2} \leq s$.

The lower bound on the interval we give should certainly be improved, but let us point that this would need a different method, as, to improve significantly the bound in Lemma 
3.12 , we would need to replace $G$ by a set where the expansions do not contain $2 *-$, and such a set does not contain elements below $\frac{1}{2}$.

3.6. The lower BL spectrum. Proposition 3.8 allows us to compute $\mathcal{B}^{\prime}$ for individual interval exchanges, by the usual trick of replacing the 1 in the numerators by the quantity in relation (11), thus we have to estimate ratios of lengths and frequencies; for example, for $m_{k}=n_{k}=2$ and $\eta_{k} \eta_{k+1}=-$, we have $l_{1, o_{k}}=l_{2, o_{k}}, r_{1, o_{k}}=r_{2, o_{k}} ; \mathcal{B}^{\prime}$ is the smallest of the lower limits (taken for $\eta_{k}=-$ ) of $\frac{1}{\Delta_{k} \mid w_{2, o_{k-1}+m_{k}-1}}, \frac{1}{l_{2, o_{k}}\left|w_{2, o_{k-1}+m_{k} \mid}\right|}, \frac{1}{r_{2, o_{k}} z_{k}}$; taking into account that $\left|M_{2, o_{k-1}}\right|=\left|M_{1, o_{k-1}}\right|$, we check that these three quantitites are all equal and that $\mathcal{B}^{\prime}=5-\sqrt{5}=2,763 \ldots$

But if we want to compute $\mathcal{B}^{\prime}$ for a general three-interval exchange, these formulas are complicated and do not reduce to simpler expressions as for $\mathcal{B}$. However, the lower BL spectrum for the family is fully known, and quite different from the lower BL spectrum of rotations.

Theorem 3.14. The lower BL spectrum of the family of three-interval exchanges is the interval $[2,+\infty]$.

\section{Proof}

No number smaller than 2 can be in the lower BL spectrum because of Lemma 1.2. We take $2 \leq s \leq+\infty$, and choose integers $m_{k}<n_{k}$ such that $m_{k}$ and $n_{k}$ grow to infinity with $k$, and $\frac{m_{k}+n_{k}}{m_{k}} \rightarrow s$ when $k$ goes to infinity; we require $n_{k}-m_{k} \rightarrow+\infty$, which is an additional condition when $s=2$; we choose $\eta_{k}=-$ for all $k$.

Then by Lemmas 3.3 and 3.4, we have $\left|w_{2, o_{k-1}+m_{k}-1}\right| \leq\left|w_{2, o_{k-1}+m_{k}}\right| \leq\left|w_{1, o_{k-1}+n_{k}-1}\right| \leq$ $\left|w_{1, o_{k-1}+n_{k}}\right|, l_{2, o_{k}} \leq r_{1, o_{k}} \leq r_{2, o_{k}}, l_{2, o_{k}} \leq l_{1, o_{k}} \leq r_{2, o_{k}}$. By Proposition 3.8, $\mathcal{B}^{\prime}$ is the smaller of the lower limits of $\frac{1}{\Delta_{k} \mid w_{2, o_{k-1}+m_{k}-1 \mid}}$ and $\frac{1}{l_{2, o_{k}} z_{k}}$.

Now $\left|w_{2, o_{k-1}+m_{k}-1}\right|$ is equivalent to $m_{k} z_{k-1}$ while, by the computations in the proof of Proposition $3.7 \frac{1}{\Delta_{k} z_{k-1}}$ is close to $v_{k}$ (because $t_{k} \rightarrow 0$ ) which is equivalent to $m_{k}+n_{k}$, thus the first lower limit is a limit and is equal to $s$. The same is true for the second one as $\frac{1}{\Delta_{k+1} z_{k}}$ and $\frac{\Delta_{k}}{\Delta_{k+1}}$ are equivalent to $m_{k+1}+n_{k+1}$, and, because $r_{1, o_{k}}+r_{2, o_{k}}$ is equivalent to $\Delta_{k}$ and $r_{1, o_{k}}-r_{2, o_{k}}$ is equivalent to $\left(m_{k+1}-n_{k+1}\right) \Delta_{k+1}$, we get that $\frac{l_{2, o_{k}}}{\Delta_{k}}$ is equivalent to $\frac{m_{k+1}}{m_{k+1}+n_{k+1}}$.

Thus for some uniquely ergodic three-interval exchange transformations we have $n e_{n} \rightarrow 0$ when $n$ tends to infinity; this result, and its consequence that Boshernitzan's criterion is not a necessary condition in this family of systems, are stated without proof in [27]. Note that the covering number by intervals (see the opening of Section 2 above) of a three-interval exchange is shown in [5] to be the same as for the inducing rotation, and thus is not equal to $\frac{1}{\mathcal{B}^{\prime}}$, in contrast with the case of rotations.

\section{Arnoux-Rauzy systems}

The Arnoux-Rauzy systems are defined in [3] as the minimal symbolic systems on the alphabet $\{1,2,3\}$ such that the complexity of the language is $2 n+1$ for all $n$, and, for all $n$, there are one right special and one left special word. Then [3] proceeds to give a constructive (additive) algorithm to generate them with three families of words, built with 
three rules denoted by $a, b$ and $c$; [11] gives a multiplicative version of this construction, which we take here as a definition, valid up to permutations of $\{1,2,3\}$ : the $k_{n}$ are the number of consecutive times a given rule is used, while the $n_{i}>1$ mark the times where three consecutive rules are all different, such as, up to permutations of $\{a, b, c\}$, rule $a$ used $k_{n_{i}-1}$ times, then rule $b$ used $k_{n_{i}}$ times, then rule $c$ used $k_{n_{i}+1}$ times.

Definition 4.1. Given two infinite sequences of integers $k_{n} \geq 1, n \geq 1$, and $n_{1}<n_{2} \ldots<$ $n_{i}<\ldots$ the Arnoux-Rauzy system $\left(X_{L}, S\right)$ defined by them is the symbolic system associated to the language $L$ of all factors of $\left(H_{n}\right)_{n \in \mathbb{N}}$, where the three words $H_{n}, G_{n}, J_{n}$ are built from $H_{0}=1, G_{0}=2, J_{0}=3$ by two families of rules:

- if $n+1=n_{i}$ for some $i, H_{n+1}=G_{n} H_{n}^{k_{n+1}}, G_{n+1}=J_{n} H_{n}^{k_{n+1}}, J_{n+1}=H_{n}$;

- otherwise, $H_{n+1}=G_{n} H_{n}^{k_{n+1}}, G_{n+1}=H_{n}, J_{n+1}=J_{n} H_{n}^{k_{n+1}}$.

Every Arnoux-Rauzy system is minimal [3] and uniquely ergodic (by [7] because the complexity is $2 n+1)$. Though they are defined as symbolic systems, they have also geometric models, see [1] [2] [3][25]: every Arnoux-Rauzy system is a coding of a six-interval exchange on the circle, and some of them are codings of rotations of the 2-torus.

Proposition 4.1. Let $o_{n}=k_{1}+\ldots k_{n}, n>0$, and $o_{0}=0$. All the bispecial words of the language are the $w_{n}$, where $w_{0}$ is the empty word and for $1 \leq j \leq k_{n+1}, w_{o_{n}+j}=w_{o_{n}} H_{n}^{j}$.

For $1 \leq j \leq k_{n+1}$, let $\theta_{n, j}$, resp. $\gamma_{n, j}, \iota_{n, j}$, be the frequency of $w_{o_{n}+j} x$ where $x$ is the first letter of $H_{n+1}$, resp. $G_{n+1}, J_{n+1}$; we denote $\theta_{n, k_{n+1}}$, resp. $\gamma_{n, k_{n+1}}, \iota_{n, k_{n+1}}$, by $\theta_{n+1}$, resp. $\gamma_{n+1}$, $\iota_{n+1}$, then

- if $n+1=n_{i}$ for some $i, \theta_{n}=j \theta_{n, j}+j \gamma_{n, j}+\iota_{n, j}, \gamma_{n}=\theta_{n, j}, \iota_{n}=\gamma_{n, j}$,

- otherwise $\theta_{n}=j \theta_{n, j}+\gamma_{n, j}+j \iota_{n, j}, \gamma_{n}=\theta_{n, j}, \iota_{n}=\iota_{n, j}$.

\section{Proof}

This is a straightforward consequence of [3], taking into account the multiplicative definition, as in Proposition 9 of [11].

We check that we have the relations

$$
\begin{gathered}
\left|H_{n}\right| \theta_{n}+\left|G_{n}\right| \gamma_{n}+\left|J_{n}\right| \iota_{n}=1, \\
\left|H_{n}\right|+\left|G_{n}\right|+\left|J_{n}\right|-2\left|w_{o_{n}}\right|=3 .
\end{gathered}
$$

We shall use also Lemma 7 of [11], with the more precise (and non-trivial) estimate used in its proof: namely, for all $n$, if $\left(t_{1, n}, t_{2, n}, t_{3, n}\right)$ is the triple $\left(\left|J_{n}\right|,\left|G_{n}\right|,\left|H_{n}\right|\right)$ ordered so that $t_{1, n} \leq t_{2, n} \leq t_{3, n}$, then we have $t_{2, n} \leq t_{1, n}+t_{3, n}$.

Proposition 4.2. For an Arnoux-Rauzy system, if $S_{n}=\left|H_{n}\right|+\left|G_{n}\right|-\left|J_{n}\right|$,

$$
\begin{aligned}
& \mathcal{B}=\limsup _{i \rightarrow+\infty}\left(\frac{2\left|H_{n_{i}}\right|}{S_{n_{i}}} \frac{\theta_{n_{i}}}{\theta_{n_{i+1}+1}}+\frac{2\left|G_{n_{i}}\right|}{S_{n_{i}}} \frac{\theta_{n_{i}+1}}{\theta_{n_{i+1}+1}}+\frac{2\left|J_{n_{i}}\right|}{S_{n_{i}}}\right), \\
& \mathcal{B}^{\prime}=\liminf _{i \rightarrow+\infty}\left(\frac{2\left|H_{n_{i}}\right|}{S_{n_{i+1}}} \frac{\theta_{n_{i}}}{\theta_{n_{i+1}+1}}+\frac{2\left|G_{n_{i}}\right|}{S_{n_{i+1}}} \frac{\theta_{n_{i}+1}}{\theta_{n_{i+1}+1}}+\frac{2\left|J_{n_{i}}\right|}{S_{n_{i+1}}}\right) .
\end{aligned}
$$

\section{Proof}

On the Rauzy graph of length $m$, there are at most four frequencies, which are for example those of $w_{o_{n}+j} 1, w_{o_{n}+j} 2, w_{o_{n}+j} 3$, and $w_{o_{n}+j}$, thus $\theta_{n, j}, \gamma_{n, j}, \iota_{n, j}$ and $\theta_{n, j}+\gamma_{n, j}+\iota_{n, j}$, for the 
smallest $n$ and $j$ such that $\left|w_{o_{n}+j}\right| \geq m$. In the same way as what happens in Lemma 2.3, for $m=\left|w_{p}\right|+1$ there are only three frequencies, which are equal to the three lowest frequencies for $m=\left|w_{p}\right|$, and for $m \neq\left|w_{p}\right|+1$ all the four frequencies appear.

For all $n$, the formulas imply that $\theta_{n} \geq \gamma_{n}$ and $\theta_{n} \geq \iota_{n}$ We have always $\theta_{n+1}=\gamma_{n}$ and either $\gamma_{n+1}=\iota_{n}$ or $\iota_{n+1}=\iota_{n}$, thus always $\gamma_{n} \geq \iota_{n}$. Then for $1 \leq j \leq k_{n+1}-1$, we have either $\theta_{n, j}=\theta_{n+1}, \gamma_{n, j}=\gamma_{n+1}=\iota_{n}, \iota_{n, j}=\iota_{n+1}+\left(k_{n+1}-j\right)\left(\theta_{n+1}+\gamma_{n+1}\right)$, or $\theta_{n, j}=\theta_{n+1}$, $\iota_{n, j}=\iota_{n+1}=\iota_{n}, \gamma_{n, j}=\gamma_{n+1}+\left(k_{n+1}-j\right)\left(\theta_{n+1}+\iota_{n+1}\right)$. Hence for all $0 \leq j \leq k_{n+1}-1$, $\min \left(\theta_{n, j}, \gamma_{n, j}, \iota_{n, j}\right)=\iota_{n}$.

Taking into account that $\iota_{n+1}=\iota_{n}$ if and only if $n+1$ is not an $n_{i}$, we get that for every $i \geq 1, e_{r}$ is equal to $\iota_{n_{i}}$ from $r=\left|w_{o_{n_{i}}-1}\right|+2$ to $r=\left|w_{o_{n_{i+1}-1}}\right|+1$.

We have $\left|w_{o_{n_{i}}-1}\right|=\left|w_{o_{n_{i}}}\right|-\left|H_{n_{i}-1}\right|=\left|w_{o_{n_{i}}}\right|-\left|J_{n_{i}}\right|$, and this is $\frac{\left|H_{n_{i}}\right|+\left|G_{n_{i}}\right|-\left|J_{n_{i}}\right|}{2}$ after discarding the constant term, which gives $\mathcal{B}=\lim \sup _{i \rightarrow+\infty} \frac{2}{\iota_{n_{i}} S_{n_{i}}}$, and $\mathcal{B}^{\prime}=\liminf _{i \rightarrow+\infty} \frac{2}{\iota_{n_{i}} S_{n_{i+1}}}$. We now input the relation (19), and the fact that for any $m, \gamma_{m}=\theta_{m+1}$ and, if $m^{\prime}$ is the smallest $n_{i}>m, \iota_{m}=\iota_{m^{\prime}-1}=\gamma_{m^{\prime}}=\theta_{m^{\prime}+1}$, to get the final formulas.

Note that in the formula giving $\mathcal{B}$, the ratios of lengths of words depend only on the $n_{j}$ for $j \leq i$ and the $k_{t}$ for $t \leq n_{i}$, while the ratios of frequencies depend only on the $n_{j}$ for $j>i$ and the $k_{t}$ for $t>n_{i}$; in the formula giving $\mathcal{B}^{\prime}$, there is no such dichotomy. To every ArnouxRauzy system is associated an algoritm of simultaneous approximation of two irrationals, see [3] [11]; this involves the $\theta_{n}$ and the $\left|H_{n}\right|$, and the approximation is best when the $k_{n}$ are large; this algorithm is hidden in all the computations of this section, though only $\mathcal{B}$ is linked to the quality of the approximation, and only in a loose way.

Proposition 4.3. The upper BL spectrum of the family of Arnoux-Rauzy systems contains $+\infty$, which is reached if and only if the $k_{n}, n \in \mathbb{N}$, or the $n_{i+1}-n_{i}, i \geq 1$, are unbounded. Its smallest element, and the only one below $\frac{181}{21}=8,619 \ldots$, is reached for the Tribonacci system where $n_{i}=i$ for all $i \geq 1$ and $k_{n}=1$ for all $n \geq 1$; for this system, if $y=1,8392 \ldots$ is the root bigger than 1 of the polynomial $X^{3}-X^{2}-X-1$, then $\mathcal{B}=2 y^{2}+\frac{4 y}{y^{2}+1}=8,4445 \ldots$.

\section{Proof}

We use the estimates from [11]; if $n$ is an $n_{i}$, the smallest of the three lengths is $\left|J_{n}\right|$, and thus either $\left|H_{n_{i}}\right| \leq\left|G_{n_{i}}\right| \leq\left|H_{n_{i}}\right|+\left|J_{n_{i}}\right|$ or $\left|G_{n_{i}}\right| \leq\left|H_{n_{i}}\right| \leq\left|G_{n_{i}}\right|+\left|J_{n_{i}}\right|$, thus $\left|S_{n_{i}}\right| \leq 2\left|G_{n_{i}}\right|$ and $\left|S_{n_{i}}\right| \leq 2\left|H_{n_{i}}\right|$; thus the quantities $\frac{2\left|H_{n_{i}}\right|}{S_{n_{i}}}$ and $\frac{2\left|G_{n_{i}}\right|}{S_{n_{i}}}$ are between 1 and 2, while $\frac{2\left|J_{n_{i}}\right|}{S_{n_{i}}}$ is between 0 and 2 .

Thus the finiteness of $\mathcal{B}$ depends on the two ratios $\frac{\theta_{n_{i}}}{\theta_{n_{i+1}+1}}$ and $\frac{\theta_{n_{i}+1}}{\theta_{n_{i+1}+1}}$, and this gives the assertion on the highest value.

For the Tribonacci system, we get $\left|H_{n+1}\right|=\left|H_{n}\right|+\left|H_{n-1}\right|+\left|H_{n-2}\right|,\left|G_{n+1}\right|=\left|H_{n}\right|+\left|H_{n-1}\right|$, $\left|J_{n+1}\right|=\left|H_{n}\right|, \theta_{n}=\theta_{n+1}+\theta_{n+2}+\theta_{n+3}, \gamma_{n}=\theta_{n+1}, \iota_{n}=\theta_{n+2}$, and when $n$ is large $\left|H_{n+1}\right|$ is close to $y\left|H_{n}\right|$ while $\theta_{n}$ is close to $y \theta_{n+1}$, which gives the formula.

Let us now sketch the proof that in all other cases $\mathcal{B} \geq \frac{181}{21}$. We suppose first that $k_{m} \geq 3$ for infinitely many $m$; we take such an $m$; let $r=n_{i}$ be the largest $n_{i}<m, s=n_{i+1}$.

- if $m=r+1<s$ : then $\theta_{r+1} \geq \theta_{s+1}$, and $\theta_{r} \geq 3 \theta_{r+1}+\theta_{r+2}+3 \theta_{s+1} \geq 3\left(\theta_{r+2}+\theta_{r+3}\right)+$ $\theta_{r+2}+3 \theta_{s+1} \geq 10 \theta_{s+1}$; putting all our estimates together, we get $\mathcal{B} \geq 11$,

- if $r+1<m \leq s$ : then $\theta_{r+1} \geq \theta_{m-1} \geq 3 \theta_{m} \geq 3 \theta_{s+1}$, and $\theta_{r} \geq \theta_{r+1}+\theta_{r+2}+\theta_{s+1} \geq$ $\theta_{m-1}+\theta_{m}+\theta_{s+1} \geq 4 \theta_{m}+2 \theta_{s+1} \geq 6 \theta_{s+1}$ : we get $\mathcal{B} \geq 9$, 
- if $m=r+1=s$, let $q=n_{i-1}$ and suppose $q<m-2$; then $\theta_{q+1} \geq \theta_{m-1}+\theta_{m} \geq 4 \theta_{m}$, and $\theta_{q} \geq \theta_{m-2} \geq 2\left(\theta_{m-1}+\theta_{m}\right) \geq 8 \theta_{m}$, we get $\mathcal{B} \geq 12$,

- if $m=r+1=s$ and $k_{m} \geq 4$; then $\theta_{r+1} \geq \theta_{s+1}$, and $\theta_{r} \geq 4 \theta_{s}+4 \theta_{s+1}$, we get $\mathcal{B} \geq 9$.

Hence either $\mathcal{B} \geq 9$, or $k_{n}$ takes only the values $1,2,3$, with 3 possible only if $r=m-1$ and $q=m-2$ in the notations above. In this last case $\theta_{m-1} \geq 3\left(\theta_{m}+\theta_{m+1}\right)$, and $\theta_{m-2} \geq$ $\theta_{m-1}+\theta_{m}+\theta_{m+1} \geq 4\left(\theta_{m}+\theta_{m+1}\right)$. If $k_{m+1}=3$, we have $\theta_{m-1} \geq 9 \theta_{m+1}$ and, computing it from $m-1$, we get $\mathcal{B} \geq 9$. If $k_{m+1} \leq 2$, we have $\theta_{m} \leq 2 \theta_{m+1}+2 \theta_{m+2}+\theta_{m+3}$ while $\theta_{m+2} \leq \theta_{m+1}-\theta_{m+3}$, hence $\theta_{m} \leq 4 \theta_{m+1}$, and, computing it from $m-2$, we get $\mathcal{B} \geq \frac{35}{4}=8,75$.

Thus we can suppose now that the $k_{n}$ take only the values 1 and 2 ; then we can improve the estimates on the ratios of lengths, with $\frac{2\left|J_{n_{i}}\right|}{S_{n_{i}}} \geq \frac{1}{3}$, and, as the estimates from [11] imply that either $\left|S_{n_{i}}\right| \leq 2\left|G_{n_{i}}\right|-\left|J_{n_{i}}\right|$ or $\left|S_{n_{i}}\right| \leq 2\left|H_{n_{i}}\right|-\left|J_{n_{i}}\right|$, either $\frac{2\left|H_{n_{i}}\right|}{S_{n_{i}}}$ or $\frac{2\left|G_{n_{i}}\right|}{S_{n_{i}}}$ are greater than $\frac{8}{7}$; the lower bounds on the ratios $\frac{\theta_{m+1}}{\theta_{m}}$ are also improved. Thus we can prove that if for infinitely many $m$ either $k_{m}=k_{m+1}=2$, or $k_{m}=2$ when $m$ is not an $n_{i}$, or $m$ is not an $n_{i}$ and $m+1$ is not an $n_{i}, \mathcal{B} \geq \frac{181}{21}$

Similarly, further improvement of the estimates, and extensive computations, allow us to eliminate all the $k_{m}=2$ and all the $m$ which are not an $n_{i}$.

Theorem 4.4. The smallest element in the lower BL spectrum of the family of ArnouxRauzy systems is 2, and the largest is $+\infty$. Every integer greater or equal to 2 is in the lower $B L$ spectrum, and is an accumulation point, as is $+\infty$.

\section{Proof}

The smallest element is at least 2 by Lemma 1.2. We take $n_{i}=2 i$, with $k_{2 n+1}=1$ and a sequence $k_{2 n}$ growing to $+\infty$. Then we have $\left|J_{2 n}\right|<\left|G_{2 n}\right|<\left|H_{2 n}\right| .\left|H_{2 n+2}\right|=k_{2 n+2}\left(\left|H_{2 n}\right|+\right.$ $\left.\left|G_{2 n}\right|\right)+\left|H_{2 n}\right|,\left|G_{2 n+2}\right|=k_{2 n+2}\left(\left|H_{2 n}\right|+\left|G_{2 n}\right|\right)+\left|H_{2 n}\right|+\left|J_{2 n}\right|,\left|J_{2 n+2}\right|=\left|H_{2 n}\right|+\left|G_{2 n}\right|$. When $n$ tends to $+\infty, \frac{\left|G_{2 n}\right|}{\left|H_{2 n}\right|} \rightarrow 1$ and $\frac{\left|J_{2 n}\right|}{\left|H_{2 n}\right|} \rightarrow 0$, thus $\frac{\left|J_{2 n}\right|}{\left|S_{2 n+2}\right|} \rightarrow 0$ and both $\frac{\left|H_{2 n}\right|}{\left|S_{2 n+2}\right|}$ and $\frac{\left|G_{2 n}\right|}{\left|S_{2 n+2}\right|}$ are equivalent to $\frac{1}{4 k_{2 n+2}}$.

As for the ratios of frequencies, $\theta_{2 n+1}=k_{2 n+2}\left(\theta_{2 n+2}+\theta_{2 n+3}\right)+\theta_{2 n+4}=k_{2 n+2}\left(2 \theta_{2 n+3}+\right.$ $\left.\theta_{2 n+4}+\theta_{2 n+5}\right)+\theta_{2 n+4} \leq k_{2 n+2}\left(2 \theta_{2 n+3}+2 \theta_{2 n+4}\right)+\theta_{2 n+4}$, and $\theta_{2 n}=\theta_{2 n+1}+\theta_{2 n+2}+\theta_{2 n+3} \leq$ $\left(k_{2 n+2}+1\right)\left(2 \theta_{2 n+3}+2 \theta_{2 n+4}\right)+\theta_{2 n+4}$. As $\theta_{2 n+4} \leq \frac{\theta_{2 n+3}}{k_{2 n+4}}$, both $\frac{\theta_{2 n+1}}{\theta_{2 n+3}}$ and $\frac{\theta_{2 n}}{\theta_{2 n+3}}$ are smaller than $2 k_{2 n+2}\left(1+\epsilon_{n}\right)$, where $\epsilon_{n} \rightarrow 0$.

Putting everything together, we get that $\mathcal{B}^{\prime}$ is at most 2 , and thus equal to 2 .

We take now $n_{i}=i$, with a sequence $k_{n}$ tending to $+\infty$. Then for all $n$ we have $\left|J_{n}\right|<\left|G_{n}\right|<\left|H_{n}\right|, S_{n+1}=\left(2 k_{n+1}-1\right)\left|H_{n}\right|+\left|G_{n}\right|+\left|J_{n}\right|<\left(2 k_{n+1}+1\right)\left|H_{n}\right|, \theta_{n} \geq k_{n+1} \theta_{n+1} \geq$ $k_{n+1} k_{n+2} \theta_{n+2}$ and thus $\mathcal{B}^{\prime}$ is at least the lower limit of $\frac{2 k_{n+1} k_{n+2}}{2 k_{n+1}+1}$, which gives $+\infty$.

If we replace $k_{2 n+1}=1$ by $k_{2 n+1}=m$ in the first construction of the proof above, we get $\mathcal{B}^{\prime}=m+1$, for any integer $m \geq 1$.

If we replace the variable $k_{n}$ or $k_{2 n}$ in the constructions above by a constant $k$ and let $k$ tend to infinity, we get sequences in the lower spectrum tending to $+\infty$ or to $m+1$, for any integer $m \geq 1$. 
Thus we have a new, and very simple, family of counter-examples to the necessity of Boshernitzan's criterion.

Of course, there are other values in the lower spectrum than those in Theorem 4.4, and we conjecture that the lower BL spectrum of the family of Arnoux-Rauzy systems is the interval $[2,+\infty]$.

We look at some individual values of $\mathcal{B}$ and $\mathcal{B}^{\prime}$, for three examples which are codings of rotations of the 2-torus [1] [2].

For the Tribonacci system $\mathcal{B}^{\prime}=2 y+\frac{4}{y^{2}+1}=4,5911 \ldots$ In [13], various constants are computed for the Tribonacci system, including one covering number, though not by intervals (see Section 2 above), and a measure of the quality of simultaneous approximation of $\left(\frac{1}{y}, \frac{1}{y^{2}}\right)$ by rationals; none of these is equal to $\mathcal{B}$ or $\mathcal{B}^{\prime}$.

For $n_{i}=i$ and $k_{n}=2$, we get $\left|H_{n+1}\right|=2\left|H_{n}\right|+2\left|H_{n-1}\right|+\left|H_{n-2}\right|,\left|G_{n+1}\right|=\left|2 H_{n}\right|+\left|H_{n-1}\right|$, $\left|J_{n+1}\right|=\left|H_{n}\right|, \theta_{n}=2 \theta_{n+1}+2 \theta_{n+2}+\theta_{n+3}$, and when $n$ is large $H_{n+1}$ is close to $y_{0} H_{n}$ while $\theta_{n}$ is close to $y_{0} \theta_{n+1}$, if $y_{0}=2,83 \ldots$ is the root bigger than 1 of the polynomial $X^{3}-2 X^{2}-2 X-1$. We get $\mathcal{B}=2 y_{0}^{2}+\frac{4 y_{0}}{y_{0}^{2}+y_{0}+1}=16,96 \ldots, \mathcal{B}^{\prime}=2 y_{0}+\frac{4}{y_{0}^{2}+y_{0}+1}=5,99 \ldots$

For $n_{i}=2 i$ and $k_{n}=1$ for all $n$, we get $\left|H_{2 n}\right|=\left|H_{2 n-1}\right|+\left|H_{2 n-2}\right|,\left|H_{2 n-1}\right|=\left|H_{2 n-2}\right|+$ $\left|H_{2 n-3}\right|+\left|H_{2 n-4}\right|+\left|H_{2 n-5}\right|,\left|G_{2 n}\right|=\left|H_{2 n-1}\right|+\left|H_{2 n-2}\right|+\left|H_{2 n-3}\right|,\left|J_{2 n}\right|=\left|H_{2 n-1}\right|, \theta_{2 n}=$ $\theta_{2 n+1}+\theta_{2 n+2}+\theta_{2 n+3}, \theta_{2 n+1}=\theta_{2 n+2}+\theta_{2 n+3}+\theta_{2 n+5}$. Let $y_{1}=1,4516 \ldots$ be the root bigger than one of the polynomial $2 X^{3}-X^{2}-2 X-1, y_{2}=\frac{1}{y_{1}-1}=2,2143 \ldots, y_{4}=\frac{1}{y_{1}}+1=1,689 \ldots$ and $y_{3}=1,903 \ldots$ be the number bigger than one satisfying $y_{3}^{2} y_{4}=y_{3} y_{4}+y_{3}+1$. When $n$ is large, $\left|H_{2 n}\right|$ is close to $y_{1}\left|H_{2 n-1}\right|,\left|H_{2 n-1}\right|$ is close to $y_{2}\left|H_{2 n-2}\right|, \theta_{2 n}$ is close to $y_{3} \theta_{2 n+1}, \theta_{2 n+1}$ is close to $y_{4} \theta_{2 n+2}$. Then $\mathcal{B}=\frac{2 y_{1}^{2} y_{2}}{y_{1}^{2} y_{2}+y_{1}+1}\left(y_{3}^{2} y_{4}+\frac{y_{1}^{2} y_{2} y_{3}}{y_{1} y_{2}+y_{1}+1}+\frac{1}{y_{1}}\right)=11,61 \ldots$, which is a candidate for the second smallest value in the upper spectrum, and $\mathcal{B}^{\prime}=\frac{\mathcal{B}}{y_{1} y_{2}}=3,61 \ldots$

The Arnoux-Rauzy systems raise questions about rotations of the 2-torus, and we may ask what could be the BL spectra for that family of systems, but the problem is that at this time we do not know any coding which may be called natural. In Section 3 of [6], there is a discussion about what should be the properties of such a coding; in view of the present paper, by analogy with the rotations of the 1-torus, it becomes reasonable to add to these properties the boundedness of the lower BL spectrum these natural codings would define for the family of rotations of the 2-torus.

Now, if we code a rotation of the 2-torus with the Cartesian product of two partitions of the 1-torus, then the complexity is quadratic and all $\mathcal{B}$ and $\mathcal{B}^{\prime}$ are infinite by Lemma 1.2, which gives another trivial counter-example to the necessity of Boshernitzan's criterion, but this coding has never been considered as natural. The Arnoux-Rauzy systems were devised to provide codings with sub-linear complexity for rotations of the 2-torus, but this was succesful only in a limited number of cases. Still, if we consider these cases, the tentative lower BL spectrum of the family of rotations of the 2-torus seems to be quite different from the lower BL spectrum of rotations of the 1-torus: if we take an Arnoux-Rauzy system with $n_{i}=i$ and constant $k_{n}=k$, it is a coding of a rotation of the 2-torus by [2], and these give arbitrarily high values for $\mathcal{B}^{\prime}$; if $n_{i}=i$ and $k_{n}$ grows slowly (for example $k_{n} \leq \frac{1}{15} n$ ), we get an infinite $\mathcal{B}^{\prime}$ while the Arnoux-Rauzy system is shown in [11] to have two continuous eigenfunctions, and is still conjectured to be a coding of a rotation of the 2-torus. Thus it seems that even those Arnoux-Rauzy which do code rotations of the 2-torus fail to satisfy 
our new condition for being natural codings, and thus we need new ideas to find natural codings of these rotations.

\section{Questions}

N. Pytheas Fogg (private communication) asked what should be the BL spectra of the family of all uniquely ergodic symbolic systems. The upper BL spectrum contains the union of the Lagrange spectrum and $+\infty$, and it is quite possible that it is just that. The lower BL spectrum contains the lower BL spectrum of rotations, and the interval $[2,+\infty]$, but also at least the point $\frac{3}{2}$, which is the value of $\mathcal{B}^{\prime}$ for the so-called period-doubling symbolic system, whose language is generated by the fixed point of the substitution $a \rightarrow a b, b \rightarrow a a$ (N. Pytheas Fogg, unpublished).

N. Pytheas Fogg has also started to investigate the spectrum of the joint values of $\left(\mathcal{B}, \mathcal{B}^{\prime}\right)$ for rotations, see https://www.lirmm.fr/ monteil/hebergement/pytheas-fogg/BL_spectrum.pdf.

\section{REFERENCES}

[1] P. ARNOUX: Un exemple de semi-conjugaison entre un échange d'intervalles et une translation sur le tore, Bull. Soc. Math. France 116 (1988), p. 489-500.

[2] P. ARNOUX, Sh. ITO: Pisot substitutions and Rauzy fractals. Journées Montoises d'Informatique Théorique (Marne-la-Vallée, 2000), Bull. Belg. Math. Soc. Simon Stevin 8 (2001), p. 181-207.

[3] P. ARNOUX, G. RAUZY: Représentation géométrique de suites de complexité 2n+1, Bull. Soc. Math. France 119 (1991), p. 199-215.

[4] V. BERTHÉ: Fréquences des facteurs des suites sturmiennes. (French) [Frequencies of the factors of Sturmian sequences], Theoret. Comput. Sci. 165 (1996), p. 295-309.

[5] V. BERTHÉ, N. CHEKHOVA, S. FERENCZI: Covering numbers: arithmetics and dynamics for rotations and interval exchanges, J. Anal. Math. 79 (1999), p. 1-31.

[6] V. BERTHÉ, S. FERENCZI, L. ZAMBONI: Interactions between dynamics, arithmetics and combinatorics: the good, the bad, and the ugly, Algebraic and topological dynamics, Contemp. Math. 385 (2005), p. 333-364, Amer. Math. Soc., Providence, RI.

[7] M. BOSHERNITZAN: A unique ergodicity of minimal symbolic flows with linear block growth, $J$. Analyse Math. 44 (1984/85), p. 77-96.

[8] M. BOSHERNITZAN: A condition for minimal interval exchange maps to be uniquely ergodic, Duke Math. J. 52 (1985), p. 723-752.

[9] M. BOSHERNITEZAN: A condition for unique ergodicity of minimal symbolic flows, Ergodic Theory Dynam. Systems 12 (1992), p. 425-428.

[10] J. CASSAIGNE: Limit values of the recurrence quotient of Sturmian sequences. WORDS (Rouen, 1997). Theoret. Comput. Sci. 218 (1999), p. 3-12.

[11] J. CASSAIGNE, S. FERENCZI, A. MESSAOUDI: Weak mixing and eigenvalues for Arnoux-Rauzy sequences. Ann. Inst. Fourier (Grenoble) 58 (2008), p. 1983-2005.

[12] N. CHEKHOVA: Covering numbers of rotations, Theoret. Comput. Sci. 230 (2000), p. 97-116.

[13] N. CHEKHOVA, P. HUBERT, A. MESSAOUDI: Propriétés combinatoires, ergodiques et arithmétiques de la substitution de Tribonacci. (French) [Combinatorial, ergodic and arithmetical properties of the Tribonacci substitution], J. Théor. Nombres Bordeaux 13 (2001), p. 371-394.

[14] T. CUSICK, M. FLAHIVE: The Markoff and Lagrange spectra.Mathematical Surveys and Monographs, 30. American Mathematical Society, Providence, RI, 1989. x+97 pp.

[15] S. FERENCZI, C. HOLTON, L. ZAMBONI: The structure of three-interval exchange transformations I: an arithmetic study, Ann. Inst. Fourier (Grenoble) 51 (2001), p. 861-901.

[16] S. FERENCZI, C. HOLTON, L. ZAMBONI: The structure of three-interval exchange transformations II: a combinatorial description of the trajectories, J. Anal. Math. 89 (2003), p. 239-276.

[17] S. FERENCZI, C. HOLTON, L. ZAMBONI: The structure of three-interval exchange transformations III: ergodic and spectral properties, J. Analyse Math. 93 (2004), p. 103-138. 
[18] S. FERENCZI, T. MONTEIL: Infinite words with uniform frequencies, and invariant measures, in Combinatorics, automata and number theory, Encyclopedia of Mathematics and its Applications (No. 135), Cambridge University Press (2010), ISBN: 9780521515979, p. 373-409.

[19] S. FERENCZI, L.F.C. da ROCHA: A self-dual induction for three-interval exchange transformations, Dyn. Syst. 24 (2009), p. 393-412.

[20] S. FERENCZI, L. ZAMBONI, Structure of $k$-interval exchange transformations: induction, trajectories, and distance theorems, J. Anal. Math. 112 (2010), p. 289-328.

[21] S. FERENCZI, L. ZAMBONI: Languages of $k$-interval exchange transformations, Bulletin London Math. Soc. 40 (2008), p. 705-714.

[22] C. GRILLENBERGER: Constructions of strictly ergodic systems. I. Given entropy. Z. Wahrscheinlichkeitstheorie und Verw. Gebiete 25 (1972/73), p. 323-334.

[23] M. HALL Jr. On the sum and product of continued fractions. Ann. of Math. (2) 48, (1947), p. $966-993$.

[24] N. PYTHEAS FOGG: Substitutions in dynamics, arithmetics and combinatorics. Edited by V. Berth, S. Ferenczi, C. Mauduit and A. Siegel. Lecture Notes in Mathematics, 1794. Springer-Verlag, Berlin, 2002. xviii+402 pp. ISBN: 3-540-44141-7

[25] G. RAUZY: Nombres algébriques et substitutions, Bull. Soc. Math. France 110 (1982), p. 147-178.

[26] W. A. VEECH: Boshernitzan's criterion for unique ergodicity of an interval exchange transformation, Ergodic Theory Dynam. Systems 7 (1987), p. 149-153.

[27] W. A. VEECH: Measures supported on the set of uniquely ergodic directions of an arbitrary holomorphic 1-form. Ergodic Theory Dynam. Systems 19 (1999), p. 1093-1109.

Institut de Mathématiques de Luminy, CNRS - UMR 6206, Case 907, 163 AV. DE Luminy, F13288 Marseille Cedex 9 (France), and FÉdération de Recherche des Unités de Mathématiques de MARseille, CNRS - FR 2291

E-mail address: ferenczi@iml.univ-mrs.fr 